TRANSACTIONS OF THE

AMERICAN MATHEMATICAL SOCIETY

Volume 352, Number 2, Pages 485-513

S 0002-9947(99)02538-6

Article electronically published on October 5, 1999

\title{
EXISTENCE AND UNIQUENESS OF RECTILINEAR SLIT MAPS
}

\author{
CARL H. FITZGERALD AND FREDERICK WEENING
}

\begin{abstract}
We consider a generalization of the parallel slit uniformization in which the angle of inclination of each image slit is assigned independently. Koebe proved that for domains of finite connectivity there is, up to a normalization, a unique rectilinear slit map achieving any given angle assignment. Koebe's theorem is partially extended to domains of infinite connectivity. A uniqueness result is shown for domains of countable connectivity and arbitrary angle assignments, and an existence result is proved for arbitrary domains under the assumption that the angle assignment is continuous and has finite range. In order to prove the existence result a new extremal length tool, called the crossing-module, is introduced. The crossing-module allows greater freedom in the family of admissible arcs than the classical module. Several results known for the module are extended to the crossing-module. A generalization of Jenkins' $\theta$ module condition for the parallel slit problem is given for the rectilinear slit problem in terms of the crossing-module and it is shown that rectilinear slit maps satisfying this crossing-module condition exist.
\end{abstract}

\section{Introduction}

The theory of conformal uniformization attempts to generalize the Riemann Mapping Theorem to multiply connected domains. The goal is to provide theorems which allow the one-to-one, conformal mapping of a given domain onto a domain with prescribed geometrical properties, and to determine conditions under which such mappings are uniquely determined. A classical example is the parallel slit uniformization. This uniformization is based on a theorem due to Grötzsch [Gr1] and de Possel $[\mathrm{dP}]$ which shows that an arbitrary domain can be mapped conformally onto a domain all of whose complementary components are parallel slits (i.e., straight line segments inclined at a fixed angle or points). Grötzsch [Gr2] showed that requiring a certain extremal length condition on the image produces a unique normalized map. Jenkins $[\mathrm{J}]$ later reformulated this condition to obtain the following theorem.

Theorem 1.1 (Grötzsch and Jenkins). If $\Omega$ is an arbitrary domain containing $\infty$, then for each angle $\theta \in[0, \pi)$ there exists a unique parallel slit map $g_{\theta}(z)$ with expansion

$$
g_{\theta}(z)=z+\frac{a_{1}}{z}+\cdots \quad \text { near } \infty
$$

Received by the editors July 3, 1995 and, in revised form, October 24, 1996 and February 19, 1999.

1991 Mathematics Subject Classification. Primary 30C35; Secondary 30C20, 31A15.

Key words and phrases. Conformal uniformization, slit maps, extremal length.

(C)1999 American Mathematical Society 
that satisfies the following condition.

$$
\left\{\begin{array}{l}
\text { For any sufficiently large square domain } S_{\theta} \text {, oriented symmetrically } \\
\text { about the origin with sides at inclination } \theta \text { and } \theta+\pi / 2 \text {, the module } \\
\text { of the family of arcs in } S_{\theta} \cap g_{\theta}(\Omega) \text { which join the sides of } S_{\theta} \text { at } \\
\text { inclination } \theta+\pi / 2 \text { is equal to one. }
\end{array}\right.
$$

In particular this theorem shows that normalized parallel slit maps are uniquely determined on domains of countable connectivity, but not in general on infinitely connected domains without requiring a condition such as $\left(\mathrm{P}_{\theta}\right)$ (For instance, if $E$ is a Cantor set of positive measure on $[0,1]$ and $\Omega$ is the domain whose complement consists of the horizontal slits $\{x+i y: x \in[0,1], y \in E\}$, then both the identity and $g_{0}(z)$ are horizontal slit maps on $\Omega$.)

The rectilinear slit uniformization generalizes the parallel slit uniformization by allowing the angle of inclination of each image slit to be assigned independently. The main results of this paper can be summarized as follows.

- Normalized rectilinear slit maps are shown to be uniquely determined on domains of countable connectivity (see Theorem 2.3).

- An example of a domain of countable connectivity and a (discontinuous) angle assignment for which no normalized rectilinear slit map achieving the assignment exists is demonstrated (see the end of section 2).

- A new extremal length tool called the crossing-module is developed. The crossing-module extends the definition of the classical module to allow arcs to cross over specified boundary components of the domain. Useful results regarding the cross-module of a domain whose outer boundary component is a rectangle are obtained (see Theorems 3.3 and 3.6).

- The crossing-module is used to state an extremal length condition, called $\left(\mathrm{NP}_{\Theta}\right)$, which generalizes Jenkins' $\left(\mathrm{P}_{\theta}\right)$ condition in the case of an angle assignment which is continuous and has finite range (see Theorem 4.4).

- For an arbitrary domain it is shown that there exists a rectilinear slit map achieving a given continuous angle assignment of finite range which satisfies the $\left(\mathrm{NP}_{\Theta}\right)$ condition (see Theorem 4.4).

In $[\mathrm{W}]$ it was conjectured that condition $\left(\mathrm{NP}_{\Theta}\right)$ determined a unique rectilinear slit map. This conjecture has recently been shown to be true by Maitani and Minda $[\mathrm{MM}]$.

In the remainder of this section we give some precise definitions and provide some of the history of the rectilinear slit uniformization and related uniformizations.

Definitions. Let $\Omega$ be a domain in the extended plane $\widehat{\mathbb{C}}$.

(i) The space of complementary components of $\Omega$ will be denoted by $\mathcal{C}(\Omega)$.

(ii) A mapping $\Theta: \mathcal{C}(\Omega) \rightarrow \mathbb{R}$ is called an angle assignment on $\mathcal{C}(\Omega)$. Inasmuch as $\Omega$ and $\mathcal{C}(\Omega)$ determine each other, the phrase $\Theta$ is an angle assignment on a domain $\Omega$ will sometimes be used.

(iii) If $f: \Omega \rightarrow \Omega^{\prime}$ is a homeomorphism, then we shall use the notation $f^{\mathcal{C}}$ to denote the correspondence from $\mathcal{C}(\Omega)$ to $\mathcal{C}\left(\Omega^{\prime}\right)$ induced by $f(z)$.

(iv) If $\Theta$ is an angle assignment on $\Omega$ and if $f: \Omega \rightarrow \Omega^{\prime}$ is a conformal homeomorphism such that for each $C \in \mathcal{C}(\Omega), f^{\mathcal{C}}(C)$ is a slit which lies on a line of inclination $\Theta(C)$ to the positive real axis, then $f(z)$ is said to achieve the angle assignment $\Theta$ (see Figure 1). 


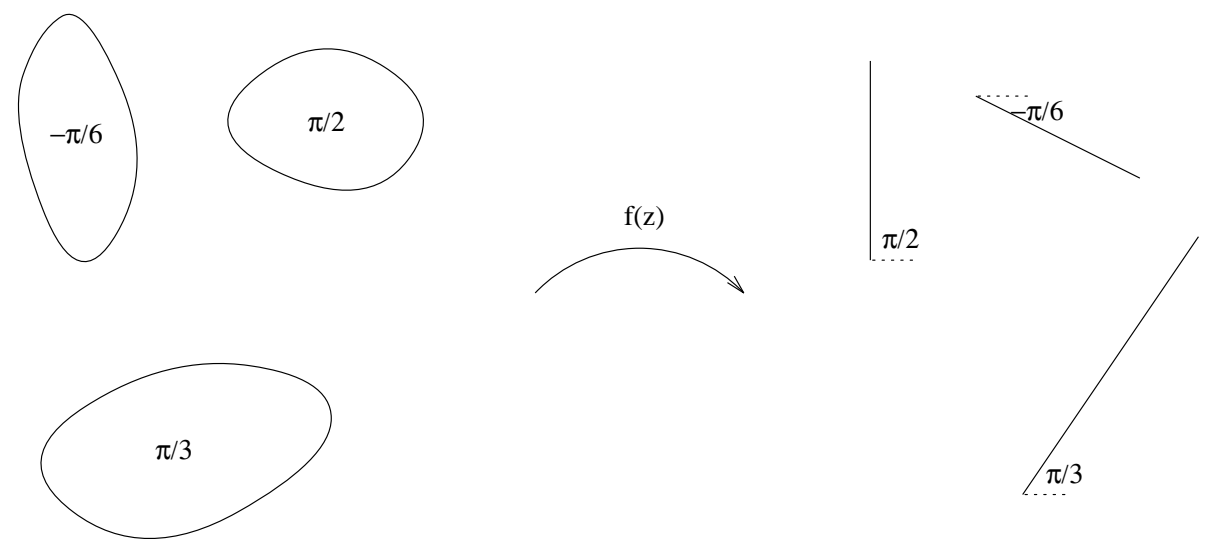

FiguRE 1. A rectilinear slit map achieving an angle assignment.

(v) A function which achieves some angle assignment is called a rectilinear slit map. The function is said to be normalized if $\infty$ belongs to the domain and the function has the expansion $z+a_{1} z^{-1}+\cdots$ near $\infty$.

For the case of domains of finite connectivity the rectilinear slit mapping problem was completely solved in 1918 by Koebe [K]. He used the continuity method to prove the following theorem.

Theorem 1.2 (Koebe). If $\Omega$ is a finitely connected domain containing $\infty$ and $\Theta$ is any angle assignment on $\mathcal{C}(\Omega)$, then there exists a unique rectilinear slit map $f(z)$ achieving $\Theta$ which satisfies the normalization

$$
f(z)=z+\frac{a_{1}}{z}+\cdots \quad \text { near } \infty .
$$

Various other uniformizations were found for domains of finite connectivity (see $[\mathrm{Go}]$ ), and ultimately very general theorems were proven. In particular, Harrington [Ha] showed that for any assignment of shapes to the boundary component of a given domain there exists a conformal map on the domain such that the boundary components in correspondence have the assigned shapes (allowing scaling but not rotation). A new proof of this theorem and certain generalizations have recently been given by Schramm in [Sc1].

Obtaining uniformization results on domains of infinite connectivity has proven to be much more difficult; for example, Koebe's circle uniformization problem while recently solved for the case of countably connected domains (see [HS]) is still open in general. Much attention has been given to slit mappings, parallel and otherwise. The original work in this area is due to Grötzsch circa 1930. In a series of articles (see $[\mathrm{J}]$ for references) he proved various existence and uniqueness results using extremal problem arguments in combination with his "method of strips" a precursor to the modern notion of extremal length.

A number of Grötzsch's theorems and proofs have been refined over the years. For instance Jenkins uses the modern formulation of extremal length to state the condition $\left(\mathrm{P}_{\theta}\right)$ of Theorem 1.1 which is not trivially equivalent to the original condition given by Grötzsch. In the case of circular and radial slit uniformization, a simple proof of existence and uniqueness was given in 1960 by Reich and Warschawski $[\mathrm{RW}]$. It is worth noting that the slit map which they found as the 
unique solution to an extremal problem also has the property that its image is a minimal slit domain in a sense analogous to that of condition $\left(\mathrm{P}_{\theta}\right)$. Later Marden and Rodin $[\mathrm{MR}]$ obtained an extremal length result on a general Riemann surface which in particular implied the results of Reich and Warschawski and provided a new characterization of uniqueness. Also notable in these uniformization problems is the approach of using principal functions to obtain various slit maps as the solutions to certain extremal problems within the framework of Riemann surfaces (see $[\mathrm{AS}],[\mathrm{NS}]$, or $[\mathrm{RS}]$ ).

Attention to the rectilinear slit mapping problem returned in the mid 1970's when Rodin began its study on domains of infinite connectivity in the hopes of obtaining insight into Koebe's circle uniformization problem. In 1977 Rodin [ABB] suggested that a continuity assumption on the angle assignment might be sufficient to imply the existence of a rectilinear slit map achieving an otherwise arbitrary assignment on any domain. Roughly speaking, an angle assignment $\Theta$ is continuous if for every sequence $\left\{C_{n}\right\}$ of complementary components which converges to a complementary component $C$, the sequence $\left\{\Theta\left(C_{n}\right)\right\}$ converges to $\Theta(C)$ modulo $\pi$. This continuity condition can be described more easily in terms of the natural topology on $\mathcal{C}(\Omega)$. Here a subset $\mathcal{O} \subset \mathcal{C}(\Omega)$ is open if and only if there exists an open set $U$ in $\widehat{\mathbb{C}}$ such that

$$
\mathcal{O}=\{C \in \mathcal{C}(\Omega): C \subset U\} .
$$

Definition. For any domain $\Omega$ in $\widehat{\mathbb{C}}$, an angle assignment $\Theta$ on $\mathcal{C}(\Omega)$ is said to be continuous provided that the map $e^{i 2 \Theta}: \mathcal{C}(\Omega) \rightarrow\{z:|z|=1\}$ is continuous.

This work shows that Rodin's continuity assumption is sufficient to imply the existence of a rectilinear slit map achieving any angle assignment with finite range. The question of existence under the continuity assumption for the case of angle assignments with infinite range is still open.

\section{Uniqueness of Rectilinear Slit Maps}

The proof of the uniqueness theorem is by the argument principle. The main difficulty is in choosing an appropriate finite collection of curves homologous to zero and a function on which to apply the argument principle. The method is motivated by He and Schramm's proof of the uniqueness of the circle normalization for domains of countable connectivity [HS]. In their proof, however, they extended maps continuously to a larger domain and applied a generalized argument principle; we need only restrict the maps to a suitable finitely connected subdomain and apply the standard argument principle.

In the course of the proof we shall use the following approximation lemmas. A proof of the first may be found in [W], while the second follows immediately from the first. Here, and elsewhere, we use the notation In $[\gamma]$ to denote the bounded domain determined by a Jordan curve $\gamma$ lying in the finite complex plane; similarly Out $[\gamma]$ will denote the unbounded domain determined by $\gamma$.

Lemma 2.1. If $\Omega$ is a domain in $\widehat{\mathbb{C}}$ containing $\infty$ and $C \in \mathcal{C}(\Omega)$, then for any $\epsilon>0$ there exists a Jordan curve $\gamma$ in $\Omega$ such that $C \subset \operatorname{In}[\gamma]$ and

$$
z \in \Omega \cap \operatorname{In}[\gamma] \quad \Rightarrow \quad \operatorname{dist}(z, C)<\epsilon
$$


Lemma 2.2. If $\Omega$ is a domain containing $\infty$ and $C \in \mathcal{C}(\Omega)$, then for any open set $U$ in $\widehat{\mathbb{C}}$ containing $C$ there exists a Jordan curve $\gamma$ in $\Omega$ such that

$$
C \subset \operatorname{In}[\gamma] \subset U \text {. }
$$

Theorem 2.3. If $\Omega$ is a countably connected domain which contains $\infty$ and $\Theta$ is any angle assignment on $\mathcal{C}(\Omega)$, then there exists at most one rectilinear slit mapping $f(z)$ achieving $\Theta$ which satisfies the normalization

$$
f(z)=z+\frac{a_{1}}{z}+\cdots \quad \text { near } \infty .
$$

Proof. Suppose to the contrary that there were two distinct normalized rectilinear slit maps $\varphi(z)$ and $\psi(z)$ achieving $\Theta$. By considering $\varphi \circ \psi^{-1}(z)$ we may reduce to the case where $\Omega$ itself is a domain with each component $C \in \mathcal{C}(\Omega)$ lying on a line of inclination $\Theta(C)$, and where there exists a normalized map $f(z) \not \equiv z$ on $\Omega$ that achieves $\Theta$. We shall show that this situation is impossible.

The difference $f(z)-z$ is analytic in $\Omega$ and has the convergent expansion

$$
\frac{a_{j}}{z^{j}}+\cdots \quad \text { near } \infty
$$

for some $j \geq 1$ and $a_{j} \neq 0$. Thus the image of a large circle under $f(z)-z$ has winding number $-j$ about the origin. This follows by Rouché's Theorem, since we may choose $R$ sufficiently large such that $\{z:|z| \geq R\} \subset \Omega$, and

$$
\left|f(z)-z-\frac{a_{j}}{z^{j}}\right|<\frac{1}{2}\left|\frac{a_{j}}{z^{j}}\right| \quad \text { on }|z|=R \text {. }
$$

In fact inequality (1) shows that as $z$ describes the circle $C_{R}=\{z:|z|=R\}$ once in the positive direction, $\arg [f(z)+a-z]$ changes by $-2 \pi j$ for any $|a|<\frac{1}{2}\left|a_{j}\right| R^{-j}$.

Let $f_{a}(z)=f(z)+a$ for any $a \in \mathbb{C}$. Since $f_{a}(z)$ is simply a translation of $f(z)$, each of the maps $f_{a}(z)$ achieve the angle assignment $\Theta$. For a fixed $a$ we imagine that the image of $f_{a}(z)$ is in the same plane as the domain $\Omega$. For any $C \in \mathcal{C}(\Omega)$, $C$ and $f_{a}{ }^{\mathcal{C}}(C)$ are parallel slits. Thus by choosing $a$ appropriately we can achieve

$$
C \cap f_{a}{ }^{\mathcal{C}}(C)=\varnothing \text { for all } C \in \mathcal{C}(\Omega)
$$

and $|a|<\frac{1}{2}\left|a_{j}\right| R^{-j}$. Indeed, since $\mathcal{C}(\Omega)$ is countable there exists $\theta_{0} \in[0, \pi)$ not in the range of $\Theta(\bmod \pi)$. If $a$ is taken to be of the form $a=r e^{i \theta_{0}}$, then for each $C \in \mathcal{C}(\Omega)$ there is (because $\left.\theta_{0} \neq \Theta(C)(\bmod \pi)\right)$ at most one value of $r$, say $r_{C}$, such that $C \cap f_{a}{ }^{\mathcal{C}}(C) \neq \varnothing$. Hence we may take $a=r_{0} e^{i \theta_{0}}$ where $\left|r_{0}\right|<\frac{1}{2}\left|a_{j}\right| R^{-j}$ and $r_{0}$ does not equal any, of the countably many, $r_{C}$.

Having fixed $a$, we now claim that there exists a finite collection $\left\{\gamma_{k}\right\}_{k=1}^{n}$ of Jordan curves in $\Omega_{R}=\Omega \cap\{z:|z|<R\}$ satisfying

$$
\begin{array}{ll}
\operatorname{In}\left[\gamma_{k}\right] \cap \operatorname{In}\left[\gamma_{l}\right]=\varnothing & \text { for } k \neq l, \\
\operatorname{In}\left[\gamma_{k}\right] \cap \operatorname{In}\left[f_{a}\left(\gamma_{k}\right)\right]=\varnothing & \text { for each } k, \text { and } \\
C \subset \bigcup \operatorname{In}\left[\gamma_{k}\right] & \text { for each } C \in \mathcal{C}(\Omega) .
\end{array}
$$

To see this observe first that since $\Omega^{c}$ is compact, if there exists a sequence $\left\{\gamma_{k}\right\}_{k=1}^{\infty}$ of Jordan curves in $\Omega_{R}$ satisfying properties (3), (4), and (5), then the sequence may be replaced by a finite collection as required. Thus, since $\mathcal{C}(\Omega)$ is countable, it suffices to show that if $\left\{\gamma_{k}\right\}_{k=1}^{m}$ possess properties (3) and (4), and $C \in \mathcal{C}(\Omega)$ is such that $C \not \subset \bigcup_{k=1}^{m} \operatorname{In}\left[\gamma_{k}\right]$, then there exists a Jordan curve $\gamma_{m+1}$ in $\Omega_{R}$ enclosing $C$ and such that $\left\{\gamma_{k}\right\}_{k=1}^{m+1}$ possess properties (3) and (4). 
Suppose that $\left\{\gamma_{k}\right\}_{k=1}^{m}$ and $C$ are as just described. By Lemma 2.1 we may choose a Jordan curve $\sigma$ in $\Omega$ such that $C \subset \operatorname{In}[\sigma]$ and

$$
z \in \Omega \cap \operatorname{In}[\sigma] \Rightarrow \operatorname{dist}(z, C)<\frac{1}{2} \operatorname{dist}\left(C, f_{a}{ }^{\mathcal{C}}(C)\right) .
$$

Likewise there exists a Jordan curve $\sigma^{\prime}$ in $f_{a}(\Omega)$ such that $f_{a}{ }^{\mathcal{C}}(C) \subset \operatorname{In}\left[\sigma^{\prime}\right]$ and

$$
w \in f_{a}(\Omega) \cap \operatorname{In}\left[\sigma^{\prime}\right] \quad \Rightarrow \quad \operatorname{dist}\left(w, f_{a}{ }^{\mathcal{C}}(C)\right)<\frac{1}{2} \operatorname{dist}\left(C, f_{a}{ }^{\mathcal{C}}(C)\right) .
$$

The curves $\sigma$ and $\sigma^{\prime}$, thought of as lying in the same plane, satisfy $\operatorname{In}[\sigma] \cap \operatorname{In}\left[\sigma^{\prime}\right]=\varnothing$; while $\operatorname{In}[\sigma] \cap \operatorname{In}\left[f_{a}^{-1}\left(\sigma^{\prime}\right)\right] \neq \varnothing$ as $C$ belongs to the intersection (this follows since $\left.f_{a}(\infty)=\infty\right)$. Thus, the set

$$
U=\operatorname{In}[\sigma] \cap \operatorname{In}\left[f_{a}^{-1}\left(\sigma^{\prime}\right)\right] \cap\{z:|z|<R\} \cap \bigcap_{k=1}^{m} \operatorname{Out}\left[\gamma_{k}\right]
$$

is open and contains $C$. By Lemma 2.2 we may take $\gamma_{m+1}$ to be a Jordan curve in $\Omega$ such that

$$
C \subset \operatorname{In}\left[\gamma_{m+1}\right] \subset U
$$

The choice of $\sigma$ and $\sigma^{\prime}$, the fact that $f_{a}(\infty)=\infty$, and the definition of $U$ imply that $\left\{\gamma_{k}\right\}_{k=1}^{m+1}$ satisfies (3) and (4). This shows our claim.

We are now ready to apply the argument principle to the cycle

$$
\Gamma=C_{R}-\sum_{k=1}^{n} \gamma_{k} \quad \text { (all curves positively oriented) }
$$

and the function $f_{a}(z)-z$. Properties (3) and (5) of $\left\{\gamma_{k}\right\}_{k=1}^{n}$ show that $\Gamma$ is homologous to zero. We have already seen that $\arg \left[f_{a}(z)-z\right]$ changes by $-2 \pi j$ as $z$ describes $C_{R}$. Consider now the change of argument of $f_{a}(z)-z$ as $z$ describes a curve $\gamma_{k}$ for some fixed $k$. By composing with a homotopy we may, by property (4), deform the image of $\gamma_{k}$ under $f_{a}(z)$ to be a point in Out $\left[\gamma_{k}\right]$ without altering the change in argument. It is therefore clear that the change in $\arg \left[f_{a}(z)-z\right]$ is zero as $z$ describes $\gamma_{k}$. Our conclusion is that the change of argument of $f_{a}(z)-z$ on the cycle $\Gamma$ is $-2 \pi j<0$. This implies that $f_{a}(z)-z$ has a singularity in $\Omega_{R}$. However we know that $f_{a}(z)-z$ is analytic in all of $\Omega$. This contradiction completes the proof.

Remarks. 1. The assumption that the domain $\Omega$ is of countable connectivity cannot be entirely removed from the theorem. Indeed it is known that even for the parallel slit uniformization there is a lack of uniqueness in the case of uncountable connectivity [J, p. 84].

2. The connectivity assumption was used in two different ways in the proof of Theorem 2.3. First it was used in order to obtain a sufficiently small complex number $a$ such that (2) held. Then it was used to show the existence of a finite collection $\left\{\gamma_{k}\right\}_{k=1}^{n}$ of Jordan curves satisfying properties (3), (4), and (5). This latter argument can be replaced with an argument relying only on the compactness of $\Omega^{c}$.

3. In the case of a parallel assignment Jenkins' weaker condition $\left(\mathrm{P}_{\theta}\right)$ concerning the extremal length of the image, characterizes a unique parallel slit map (see Theorem 1.1). We shall formulate a generalization of this condition called $\left(\mathrm{NP}_{\Theta}\right)$ (see Theorem 4.4) for continuous angle assignments with finite range. It has recently 
been shown by Maitani and Minda $[\mathrm{MM}]$ that $\left(\mathrm{NP}_{\Theta}\right)$ implies uniqueness in the case of such a rectilinear assignment. Their proof uses the condition $\left(\mathrm{NP}_{\Theta}\right)$ to show that the rectilinear slit map has a certain boundary behavior and then they apply a uniqueness result of Shiba [Sh].

4. Our proof using the argument principle could be extended to the case where the connectivity assumption is replaced with the assumption of condition $\left(\mathrm{NP}_{\Theta}\right)$ if one could show that $\left(\mathrm{NP}_{\Theta}\right)$ is sufficient to imply the existence of a sufficiently small complex constant $a$ such that (2) holds for a domain of arbitrary connectivity.

Theorem 2.3 can be used to demonstrate an example which shows that not all angle assignments can be achieved by normalized maps. The example we provide is of a discontinuous angle assignment on a domain of countable connectivity. This shows that the existence portion of Koebe's theorem is sharp, and also motivates using Rodin's continuity assumption for proving existence.

Example. For each nonzero integer $n$, let $h_{n}$ be the horizontal segment whose imaginary part is $1 / n$ and whose real part ranges between -1 and 1 ; and let $h_{0}$ be the segment $[-1,1]$ on the real axis. Let $\Omega$ be the domain whose complement consists of the line segments $h_{n}$, and consider the angle assignment $\Theta$ on $\mathcal{C}(\Omega)$ defined by

$$
\Theta\left(h_{n}\right)= \begin{cases}0, & n \neq 0, \\ \pi / 2, & n=0 .\end{cases}
$$

Claim. No normalized mapping can achieve the assignment $\Theta$ on $\mathcal{C}(\Omega)$.

The proof of this claim uses the uniqueness theorem in two different ways: first to conclude that the image of a supposed map achieving the assignment has a certain symmetry, and second to rule out one possible form of the image. With regard to the latter use, we remind the reader that a point component is a slit whose angle of inclination may be taken to be any desired real number.

Proof of Claim. Suppose to the contrary that $f(z)$ achieves $\Theta$ and is normalized with expansion $z+a_{1} z^{-1}+\cdots$ near $\infty$. The uniqueness theorem and the symmetry of $\Omega$ imply that $f(z)=-\overline{f(-\bar{z})}$. It follows that for each $n \neq 0, f^{\mathcal{C}}\left(h_{n}\right)$ is a horizontal line segment symmetric to the imaginary axis. Further $f^{\mathcal{C}}\left(h_{0}\right)$ is either (i) the point $\{0\}$, or (ii) a vertical line segment $\{w: \operatorname{Re} w=0,|\operatorname{Im} w| \leq \rho\}$ for some $\rho>0$. The first case is impossible since then both the maps $f(z)$ and the identity would achieve the assignment $\widetilde{\Theta} \equiv 0$, contradicting Theorem 2.3. In the second case, by considering boundary correspondence, we deduce that if $w \rightarrow \zeta \in f^{\mathcal{C}}\left(h_{0}\right)$ through $\{w: \operatorname{Re} w<0\}$ then $f^{-1}(w) \rightarrow-1$. By the reflection principle this implies that $f^{-1}(w)$ is a constant, also impossible. Since both cases lead to contradictions, we conclude that no such $f(z)$ exists.

\section{A Generalized Module Technique}

In this section we describe a variation of the classical module which generalizes the standard notion to allow greater freedom in the family of arcs. A new module-like quantity, called the crossing-module, will be defined in Section 3.1. The crossing-module shall not be a conformal invariant; however useful estimates for the variation of quantities related to the crossing-module under a conformal map will be obtained in Section 3.2. Explicit calculation of the crossing-module for 


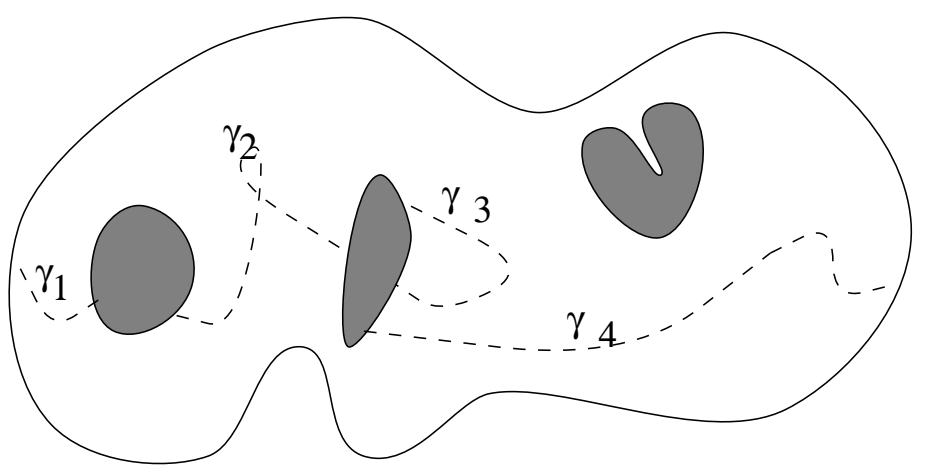

Figure 2. A chain of arcs in a domain.

a certain type of arc family will be given in Section 3.3. The crossing-module is the key tool used in Section 4 to prove the existence of rectilinear slit maps.

Recently Schramm [Sc2] has introduced a different (but similar) generalization which he calls the transboundary extremal length. His notion is a conformal invariant, and he has developed a general theory in which the transboundary extremal length is used to prove many types of uniformizations. His theory is suited toward proving the existence of uniformizations in which the complementary components of the image are all "fat" sets. It does not apply to our mapping problem because a slit is the antithesis of a fat set.

3.1. The crossing-module. The crossing-module is motivated as an attempt to develop a tool to show that some of the complementary components of a domain are slits lying at the same inclination, while other components are known not to be slits at this inclination. Essentially one would like to ignore the fact that these other components are in the complement and allow arcs to pass through them. The behavior of such arcs in the interior of the complement is of no interest, it is only important to keep track of where the boundary is crossed.

We shall define the crossing-module for domains in the extended complex plane. A module-like quantity will be defined by modifying the standard notions of $\rho$ length and $\rho$-area. The modification of the length will be to add a term representing the Euclidean distance "jumped" for each boundary crossing. While the area will be modified by adding the Lebesgue area of all the complementary regions which may be crossed.

Definitions. Let $\Omega$ be a domain in $\widehat{\mathbb{C}}$.

(i) A rectifiable arc $\gamma:[0,1] \rightarrow \mathbb{C}$ is said to join boundary components of $\Omega$ if the interior of $\gamma$ is contained in $\Omega$ and the endpoints of $\gamma$ belong to $\partial \Omega$.

(ii) A chain in $\Omega$ is a finite sum $\sigma=\sum_{j=1}^{n} \gamma_{j}$ of $\operatorname{arcs} \gamma_{j}$ each joining boundary components of $\Omega$, and such that the terminal point of $\gamma_{j}$ and the initial point of $\gamma_{j+1}$ belong to the same boundary component of $\Omega$ for $j=1,2, \ldots, n-1$ (see Figure 2).

(iii) The crossing-number of a chain $\sigma=\sum_{j=1}^{n} \gamma_{j}$ is defined to be the number $n$ of arcs comprising $\sigma$ and will be denoted by $\mathrm{c}_{\#}(\sigma)$.

(iv) The initial and terminal points of $\sigma=\sum_{j=1}^{n} \gamma_{j}$ are defined respectively as the initial and terminal points of $\gamma_{1}$ and $\gamma_{n}$. 
(v) The space of boundary components of $\Omega$ will be denoted by $\mathcal{B}(\Omega)$.

(vi) A fixed subset $\mathcal{B}$ of $\mathcal{B}(\Omega)$ is called a set of crossing-boundaries provided that the Lebesgue area of the complementary components of $\Omega$ corresponding to the elements of $\mathcal{B}$ is finite. This area will be denoted by $A_{b}(\mathcal{B})$.

(vii) If $\mathcal{B}$ is a set of crossing-boundaries, then the set of all chains in $\Omega$ which, aside for initial and terminal points, are contained in $\Omega \cup(\bigcup \mathcal{B})$ will be denoted by $C_{\Omega}(\mathcal{B})$.

(viii) A non-negative function $\rho(z)$ defined on $\Omega$ is called a metric on $\Omega$ provided that $\rho(z)$ is lower semi-continuous.

(ix) If $\rho(z)$ is a metric on $\Omega$, and $\Gamma$ is a family of chains contained in $C_{\Omega}(\mathcal{B})$, then the $\rho$-length of a chain $\sigma=\sum_{j=1}^{n} \gamma_{j} \in \Gamma$ is defined by

$$
l_{\rho}(\sigma)=\sum_{j=1}^{n} \int_{\gamma_{j}} \rho(z)|d z|+\sum_{j=1}^{n-1}\left|\gamma_{j+1}(0)-\gamma_{j}(1)\right| .
$$

(x) The $\rho$-length of a family $\Gamma$ is defined by

$$
L_{\rho}(\Gamma)=\inf _{\sigma \in \Gamma} l_{\rho}(\sigma) .
$$

(xi) The $\rho$-area of $\Omega$ with respect to $\mathcal{B}$ is defined by

$$
A_{\rho}(\Omega, \mathcal{B})=\iint_{\Omega} \rho(x, y)^{2} d x d y+A_{b}(\mathcal{B})
$$

for any set of crossing-boundaries $\mathcal{B}$.

(xii) If $\mathcal{B}$ is a set of crossing-boundaries of $\Omega$, then the crossing-module of a family of chains $\Gamma$ in $C_{\Omega}(\mathcal{B})$ is defined by the expression

$$
\mu_{\Omega, \mathcal{B}}(\Gamma)=\inf _{\rho} \frac{A_{\rho}(\Omega, \mathcal{B})}{L_{\rho}(\Gamma)^{2}}
$$

where the infimum is taken over all metrics $\rho(z)$ on $\Omega$, and indeterminate ratios are defined to be equal to $\infty$. When clear from context we shall use the notation $\mu(\Gamma)$.

(xiii) Sets $\Omega, \mathcal{B}$, and $\Gamma$ for which a crossing-module is defined shall be referred to collectively as a crossing-module problem; specifically as the crossing-module problem $(\Omega, \mathcal{B}, \Gamma)$.

Remarks. 1. If $\mathcal{B}=\varnothing$, then the definition of the crossing-module coincides with that of the module.

2. The crossing-module will not be a conformal invariant; we will, however, obtain a useful estimate (see Theorem 3.1) for the change in the area to lengthsquared ratio for a fixed metric under the action of a conformal map.

3. It will also be the case that the area to length-squared ratio will not be homogeneous in the metrics. This is not viewed as a deficiency, but rather as a normalization of the problem (see Theorem 3.3).

3.2. The conformal variance of the crossing-module. Let $(\Omega, \mathcal{B}, \Gamma)$ be a crossing-module problem. A conformal homeomorphism $f(z)$ on $\Omega$ which extends continuously to the crossing-boundaries $B \in \mathcal{B}$ induces a natural crossing-module problem 
$\left(\Omega^{f}, \mathcal{B}^{f}, \Gamma^{f}\right)$ where

$$
\begin{aligned}
\Omega^{f} & =f(\Omega), \\
\mathcal{B}^{f} & =\{f(B): B \in \mathcal{B}\}, \text { and } \\
\Gamma^{f} & =\left\{\sigma^{f}=\sum_{j=1}^{n} f\left(\gamma_{j}\right): \sigma=\sum_{j=1}^{n} \gamma_{j} \in \Gamma\right\} .
\end{aligned}
$$

The crossing-modules $\mu\left(\Gamma^{f}\right)$ and $\mu(\Gamma)$ are clearly equal when $f(z)$ is a translation or rotation, but in general they need not be equal. The following theorem provides an estimate for the difference

$$
\frac{A_{\rho^{f}}\left(\Omega^{f}, \mathcal{B}^{f}\right)}{L_{\rho^{f}}\left(\Gamma^{f}\right)^{2}}-\frac{A_{\rho}(\Omega, \mathcal{B})}{L_{\rho}(\Gamma)^{2}}
$$

where $\rho(z)$ is any metric on $\Omega$ and $\rho^{f}(w)$ is the induced metric on $f(\Omega)$ defined by

$$
\rho^{f}(w)=\rho\left(f^{-1}(w)\right)\left|f^{\prime}\left(f^{-1}(w)\right)\right| .
$$

Theorem 3.1. Let $(\Omega, \mathcal{B}, \Gamma)$ be a crossing-module problem, and $f(z)$ be a conformal homeomorphism on $\Omega$ which extends continuously to $\cup \mathcal{B}$. Assume that there exists an integer $N$ such that each $\sigma \in \Gamma$ has crossing-number at most $N$. If there exists $\epsilon>0$ such that for each $B \in \mathcal{B}$

$$
z_{1}, z_{2} \in B \quad \Rightarrow \quad|| f\left(z_{1}\right)-f\left(z_{2}\right)|-| z_{1}-z_{2}||<\epsilon,
$$

then for each metric $\rho(z)$ on $\Omega$,

$$
\left|L_{\rho^{f}}\left(\Gamma^{f}\right)-L_{\rho}(\Gamma)\right| \leq \epsilon N .
$$

If additionally there exists $\epsilon^{\prime}>0$ such that

$$
\left|A_{b}\left(\mathcal{B}^{f}\right)-A_{b}(\mathcal{B})\right|<\epsilon^{\prime}
$$

then for each metric $\rho(z)$ on $\Omega$ such that $L_{\rho}(\Gamma)>\epsilon N$,

$$
\left|\frac{A_{\rho^{f}}\left(\Omega^{f}, \mathcal{B}^{f}\right)}{L_{\rho^{f}}\left(\Gamma^{f}\right)^{2}}-\frac{A_{\rho}(\Omega, \mathcal{B})}{L_{\rho}(\Gamma)^{2}}\right| \leq \frac{1}{\left(L_{\rho}(\Gamma)-\epsilon N\right)^{2}}\left[\epsilon^{\prime}+\epsilon N\left(2 L_{\rho}(\Gamma)+\epsilon N\right) \frac{A_{\rho}(\Omega, \mathcal{B})}{L_{\rho}(\Gamma)^{2}}\right] .
$$

Proof. Assume that (7) holds for some $\epsilon>0$ and each $B \in \mathcal{B}$. Let $\rho$ be an arbitrary metric on $\Omega$. For a given $\sigma=\sum_{j=1}^{n} \gamma_{j} \in \Gamma$, denote the initial and terminal points of each $\gamma_{j}$ by $a_{j}$ and $b_{j}$ respectively. Since $\int_{f\left(\gamma_{j}\right)} \rho^{f}(w)|d w|=\int_{\gamma_{j}} \rho(z)|d z|$ for each $j$, it follows from the definition of $\rho$-length that

$$
l_{\rho^{f}}\left(\sigma^{f}\right)-l_{\rho}(\sigma)=\sum_{j=1}^{n-1}\left(\left|f\left(a_{j+1}\right)-f\left(b_{j}\right)\right|-\left|a_{j+1}-b_{j}\right|\right) .
$$

Thus

$$
L_{\rho^{f}}\left(\Gamma^{f}\right) \leq l_{\rho^{f}}\left(\sigma^{f}\right) \leq l_{\rho}(\sigma)+\sum_{j=1}^{n-1}\left(\left|f\left(a_{j+1}\right)-f\left(b_{j}\right)\right|-\left|a_{j+1}-b_{j}\right|\right),
$$

and the assumption (7) yields that

$$
L_{\rho f}\left(\Gamma^{f}\right) \leq l_{\rho}(\sigma)+\epsilon N .
$$


Since this is true for any $\sigma \in \Gamma$, it follows that

$$
L_{\rho^{f}}\left(\Gamma^{f}\right)-L_{\rho}(\Gamma) \leq \epsilon N .
$$

Similarly, starting from equation (11), one can show that

$$
L_{\rho}(\Gamma)-L_{\rho^{f}}\left(\Gamma^{f}\right) \leq \epsilon N .
$$

Hence (8) holds.

Now assume additionally that (9) holds for some $\epsilon^{\prime}>0$. To simplify calculations define

$$
A=\iint_{f(\Omega)} \rho^{f}(u, v)^{2} d u d v=\iint_{\Omega} \rho(x, y)^{2} d x d y .
$$

The definition of $\rho$-area and elementary algebra implies that

$$
\begin{aligned}
& \frac{A_{\rho^{f}}\left(\Omega^{f}, \mathcal{B}^{f}\right)}{L_{\rho^{f}}\left(\Gamma^{f}\right)^{2}}-\frac{A_{\rho}(\Omega, \mathcal{B})}{L_{\rho}(\Gamma)^{2}} \\
& =\frac{A+A_{b}\left(\mathcal{B}^{f}\right)}{L_{\rho^{f}}\left(\Gamma^{f}\right)^{2}}-\frac{A+A_{b}(\mathcal{B})}{L_{\rho}(\Gamma)^{2}} \\
& =\frac{1}{L_{\rho^{f}}\left(\Gamma^{f}\right)^{2}}\left[A+A_{b}\left(\mathcal{B}^{f}\right)-\left(A+A_{b}(\mathcal{B})\right)+\left(1-\frac{L_{\rho^{f}}\left(\Gamma^{f}\right)^{2}}{L_{\rho}(\Gamma)^{2}}\right)\left(A+A_{b}(\mathcal{B})\right)\right] \\
& =\frac{1}{L_{\rho^{f}}\left(\Gamma^{f}\right)^{2}}\left[A_{b}\left(\mathcal{B}^{f}\right)-A_{b}(\mathcal{B})\right. \\
& \left.\quad+\left(\frac{\left(L_{\rho}(\Gamma)-L_{\rho^{f}}\left(\Gamma^{f}\right)\right)\left(L_{\rho}(\Gamma)+L_{\rho^{f}}\left(\Gamma^{f}\right)\right)}{L_{\rho}(\Gamma)^{2}}\right) A_{\rho}(\Omega, \mathcal{B})\right] .
\end{aligned}
$$

Inequality (10) follows from this equation, under the assumption that $L_{\rho}(\Gamma)-\epsilon N>$ 0 , by taking absolute values and using inequalities (8) and (9).

Remarks. 1. The important feature of estimate (10) to note is that the right hand side depends only on the original crossing-module problem and on $\epsilon$ and $\epsilon^{\prime}$. Moreover it tends to zero as $\epsilon$ and $\epsilon^{\prime}$ tend to zero.

2. Theorem 3.1 can be used to obtain a lower bound for $\mu(\Gamma)$ even when there is no bound to the crossing-numbers of chains in $\Gamma$. One considers the subfamily $\Gamma^{N}=\left\{\sigma \in \Gamma: c_{\#}(\sigma) \leq N\right\}$; since $\Gamma^{N} \subset \Gamma$ it follows that $\mu(\Gamma) \geq \mu\left(\Gamma^{N}\right)$.

The most natural setting in which to apply Theorem 3.1 is when the conformal map $f(z)$ is close to the identity on the crossing-boundaries. This indeed will be the case in our application in Section 4. In this case, the choice of $\epsilon$ in (7) is clear. The choice of $\epsilon^{\prime}$ in (9) is less obvious. We now state a lemma which estimates how small $\epsilon^{\prime}$ may be taken in (9) under the assumption that there are only finite many crossing-boundaries each of which is a rectifiable Jordan curve.

Lemma 3.2. Let $\Omega$ be a domain and suppose that $\mathcal{B} \subset \mathcal{B}(\Omega)$ is a finite collection $\left\{B_{1}, B_{2}, \ldots, B_{n}\right\}$ of rectifiable Jordan curves. Suppose that $f(z)$ is a conformal homeomorphism on $\Omega$ which extends continuously to $\bigcup \mathcal{B}$. If $|f(z)-z|<\epsilon$ on $\bigcup \mathcal{B}$ and $\epsilon<L_{k}=\operatorname{arclength}\left(B_{k}\right)$ for each $k=1,2, \ldots, n$, then

$$
\left|A_{b}\left(\mathcal{B}^{f}\right)-A_{b}(\mathcal{B})\right|<8 \pi L \cdot \epsilon
$$

where $L=\sum_{k=1}^{n} L_{k}$. 
Proof. It suffices to assume that $n=1$. Let $\varphi(t)$ parameterize $B_{1}$ by arc length on $[0, L]$ and extend $\varphi(t)$ periodically. Consider, for each $m=1,2, \ldots,\lceil L / \epsilon\rceil$ the disk $\Delta_{m}$ of radius $2 \epsilon$ and center $\varphi(m \epsilon)$. Since any point within $\epsilon$ of $B_{1}$ is within $2 \epsilon$ of one of these centers, $f\left(B_{1}\right)$ is contained in the union of these disks. Hence it follows that

$$
\left|A_{b}\left(\mathcal{B}^{f}\right)-A_{b}(\mathcal{B})\right| \leq \sum_{m=1}^{\lceil L / \epsilon\rceil} \operatorname{area}\left(\Delta_{k}\right)<\left(\frac{L}{\epsilon}+1\right) \pi(2 \epsilon)^{2}=4(L+\epsilon) \pi \epsilon<8 \pi L \cdot \epsilon .
$$

3.3. The crossing-module of a punctured rectangular domain. Recall that the module of a rectangle (i.e., of the arcs joining a pair of opposite sides of a rectangular domain) is equal to the ratio of the length of the sides not being joined to the length of the joined sides. Further recall that this result still holds if the rectangular domain is replaced by a rectangular domain minus a collection of parallel slits, whose projection onto a line parallel to the sides joined has linear measure zero. These results are generalized by allowing the rectangular domain to have complementary components over which arcs are allowed to cross. Theorem 3.3 will show that the resulting crossing-module is equal to the appropriate ratio of the side lengths of the rectangle and that the only extremal metric is $\rho(z) \equiv 1$.

At least as important to our application is an analysis of the case in which the described domain has some boundary component that is not a crossing-boundary and which is also not a slit in the preferred direction. An explicit metric is constructed to show that the crossing-module in this case is strictly less than the relevant ratio of the side lengths of the rectangle, see Theorem 3.6.

We begin by making some definitions. Since the crossing-module is invariant under translations and rotations, we may restrict our consideration to rectangular domains whose boundary sides are parallel and symmetric to the axes.

Definitions. Let

$$
R_{a, b}=\{z:|\operatorname{Re} z|<a / 2,|\operatorname{Im} z|<b / 2\} \quad \text { for } a, b>0 .
$$

(i) A domain $\Omega$ is said to be a punctured rectangular domain if there exists a domain $D$ such that

$$
\Omega=R_{a, b} \cap D \quad \text { and } \quad D^{c} \subset R_{a, b}
$$

for some $a, b>0$; see Figure 3 . The numbers $a, b$ are called respectively the length and height of $\Omega$.

(ii) For a fixed $a$ and $b$, let $\Omega$ be a punctured rectangular domain of length $a$ and height $b$ and suppose that $(\Omega, \mathcal{B}, \Gamma)$ is a crossing-module problem. If the initial and terminal points of every $\sigma \in \Gamma$ lie on opposite vertical sides of $\partial R_{a, b}$, then $\Gamma$ is said to join the vertical sides of $\Omega$.

(iii) In the same situation, the crossing-module problem is said to be horizontally sectionable if for almost every $y \in(-b / 2, b / 2)$ there exists a chain $\sigma_{y} \in \Gamma$ joining the vertical sides of $\Omega$, whose image is $\Omega \cap\{z: \operatorname{Im} z=y\}$. Such a chain $\sigma_{y}$ is called a horizontal chain.

Note that if $\Omega$ is a punctured rectangular domain of length $a$ and height $b$ that is horizontally sectionable, then the linear measure of the projection of $\partial \Omega \backslash(\cup \mathcal{B} \cup$ $\left.\partial R_{a, b}\right)$ onto the imaginary axis is zero. 


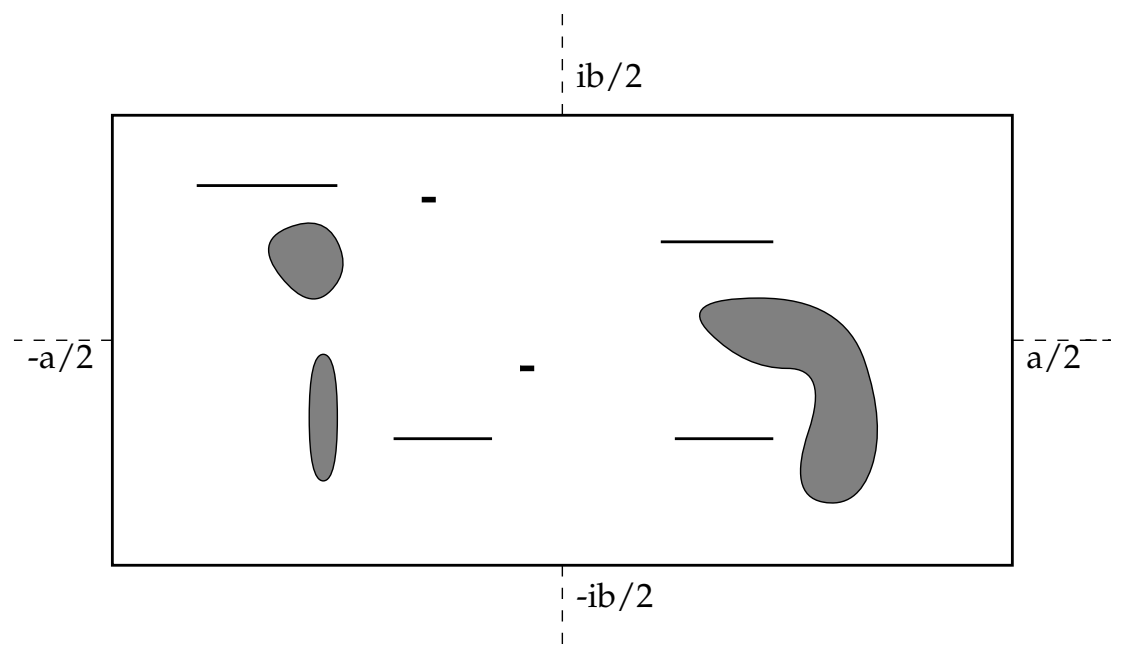

FiguRE 3. A punctured rectangular domain of length $a$ and height $b$.

Theorem 3.3. Let $\Omega$ be a punctured rectangular domain of length $a$ and height $b$. Suppose that $(\Omega, \mathcal{B}, \Gamma)$ is a crossing-module problem with $\Gamma$ joining the vertical sides of $\Omega$. If $(\Omega, \mathcal{B}, \Gamma)$ is horizontally sectionable, then

$$
\mu(\Gamma)=b / a .
$$

Further $\rho^{*}(z) \equiv 1$ is the only extremal metric provided that $\mathcal{B} \neq \varnothing$.

Proof. First note that $A_{\rho^{*}}(\Omega, \mathcal{B}) \leq a b$ and $L_{\rho^{*}}(\Gamma) \geq a$. Thus $\mu(\Gamma) \leq b / a$, and $\rho^{*}(z)$ will be shown to be extremal if the reverse inequality is shown. Let $C_{B}$ denote the complementary component corresponding to a boundary component $B \in \mathcal{B}(\Omega)$. Let $\rho(z)$ be any metric on $\Omega$, and define $\widetilde{\rho}(z)$ on $\widetilde{\Omega}=\Omega \cup\left(\bigcup\left\{C_{B}: B \in \mathcal{B}\right\}\right)$ by

$$
\widetilde{\rho}(z)= \begin{cases}\rho(z), & z \in \Omega, \\ 1, & z \in\left(\bigcup\left\{C_{B}: B \in \mathcal{B}\right\}\right) .\end{cases}
$$

Consider the family $\widetilde{\Gamma}$ consisting of all arcs $\gamma$ in $\widetilde{\Omega}$ joining the vertical sides of $\widetilde{\Omega}$ such that $\overline{\gamma \cap \Omega}$ is the image of some chain $\sigma \in \Gamma$. In other words, each chain $\sigma \in \Gamma$ is extended in all possible ways into the complementary components corresponding to the boundaries it crosses.

The definition of the metric $\widetilde{\rho}(z)$, and of area and length in a crossing-module problem imply that $A_{\rho}(\Omega, \mathcal{B})=A_{\tilde{\rho}}(\widetilde{\Omega})$ and that $L_{\rho}(\Gamma) \leq L_{\tilde{\rho}}(\widetilde{\Gamma})$. Hence

$$
\frac{A_{\rho}(\Omega, \mathcal{B})}{L_{\rho}(\Gamma)^{2}} \geq \frac{A_{\tilde{\rho}}(\widetilde{\Omega})}{L_{\tilde{\rho}}(\widetilde{\Gamma})^{2}}
$$

Since $(\Omega, \mathcal{B}, \Gamma)$ is horizontally sectionable, the horizontal segment from $-a / 2+i y$ to $a / 2+i y$ belongs to $\widetilde{\Gamma}$ for almost every $y \in(-b / 2, b / 2)$. Thus it follows from the classical theorem on the module that

$$
\frac{A_{\tilde{\rho}}(\widetilde{\Omega})}{L_{\tilde{\rho}}(\widetilde{\Gamma})^{2}} \geq b / a
$$


By combining (12) and (13), and taking the infimum over all metrics $\rho(z) \in P$ one obtains that $\mu(\Gamma) \geq b / a$ as desired.

Since it is known that the only extremal metrics in the module problem are the constants, it follows from the above extension procedure that no metric except $\rho^{*}(z)$ can be extremal.

If, with the notation of Theorem 3.3, some $B^{*} \in \mathcal{B}(\Omega) \backslash\left(\mathcal{B} \cup\left\{\partial R_{a, b}\right\}\right)$ is not a horizontal slit, then one might expect that the crossing-module would be less than $b / a$. The intuitive reason for this is that the boundary component $B^{*}$ would serve as a barrier, impeding chains from joining the vertical sides of $\Omega$. We shall show in Theorem 3.6 that this intuition is correct, under the additional assumption that the distance from $B^{*}$ to $\mathcal{B}$ is strictly positive. This theorem will then provide a test to see if a particular group of boundary components in an arbitrary domain consists of parallel slits.

A boundary component $B^{*}$ as just described can be characterized as a connected set which contains two points whose imaginary parts are different. The following lemma allows this global characterization to be transformed into a local property. Roughly speaking, the local property obtained is that there exists a square region $S$ with sides parallel to the axes and of arbitrary small length, such that within $S$ the boundary component $B^{*}$ separates a point on the left side of $S$ from all points on the right side of $S$. Additionally, this geometric property is then used to produce an inequality involving a specific metric suitable for a crossing-module problem.

By way of notation, if $S$ is the square region

$$
S=\{z: a \leq \operatorname{Re} z \leq a+s, b \leq \operatorname{Im} z \leq b+s\},
$$

then the left and right sides of $S$ are defined by

$$
(\partial S)_{l}=\{z: \operatorname{Re} z=a\} \cap \partial S \text { and }(\partial S)_{r}=\{z: \operatorname{Re} z=a+s\} \cap \partial S .
$$

Lemma 3.4. Let $D \neq \widehat{\mathbb{C}}$ be a simply connected domain containing $\infty$ such that $\partial D$ is not a horizontal slit. Then for any $s_{0}>0$,

$$
\left\{\begin{array}{l}
\text { there exists a closed square region } S \text { with sides parallel to the axes and } \\
\text { of length less than } s_{0} \text {, such that there exists a point } z_{1} \in(\partial S)_{l} \cap D, \\
\text { and such that any rectifiable arc from } z_{1} \text { to any point } z_{2} \in(\partial S)_{r} \cap D \\
\text { which is contained in } S \text { necessarily intersects } \partial D \text {. }
\end{array}\right.
$$

Moreover, for any fixed $s_{0}>0$ and square region $S$ as described in (14), there exists a disk $\Delta^{*} \subset S$ such that, if $w_{1}, w_{2} \in \partial S$ and $\gamma$ is a rectifiable arc in $D \cap S$ joining $w_{1}$ to $w_{2}$ then

$$
\int_{\gamma} \rho^{*}(z)|d z| \geq\left|\operatorname{Re} w_{1}-\operatorname{Re} w_{2}\right|
$$

where

$$
\rho^{*}(z)= \begin{cases}1, & z \in D \backslash \Delta^{*}, \\ 0, & z \in D \cap \Delta^{*} .\end{cases}
$$

So as not to interrupt the proof of Lemma 3.4 we first state the following simple result, whose proof is left to the reader, regarding the replacement of a rectifiable curve with a Jordan curve. 
Lemma 3.5. Suppose that $D$ is a domain, $K$ is a convex region, and $\gamma$ is a rectifiable arc in $D \cap K$ joining points $z_{1}$ to $z_{2}$. Then there exists a Jordan arc in $D \cap K$ joining $z_{1}$ to $z_{2}$.

Proof of Lemma 3.4. The first assertion will be proven by contradiction. Suppose that there exists $s_{0}>0$ such that

$$
\left\{\begin{array}{l}
\text { for every closed square region } S \text { with sides parallel to the axes and } \\
\text { of length less than } s_{0} \text { and every } z_{1} \in(\partial S)_{l} \cap D, \text { there exists a point } \\
z_{2} \in(\partial S)_{r} \cap D \text { and a rectifiable arc } \gamma \text { from } z_{1} \text { to } z_{2} \text { with } \gamma \subset S \\
\text { and } \gamma \cap \partial D=\varnothing .
\end{array}\right.
$$

Choose points $\zeta_{1}, \zeta_{2} \in \partial D$ with $\operatorname{Im}\left(\zeta_{1}-\zeta_{2}\right)>0$. We construct an infinite row of square regions of side length $s=\min \left\{s_{0}, \operatorname{Im}\left(\zeta_{1}-\zeta_{2}\right)\right\} / 4$ which will separate $\zeta_{1}$ from $\zeta_{2}$. Define $b=\operatorname{Im}\left(\zeta_{1}+\zeta_{2}\right) / 2-s / 2$, and let

$$
S_{k}=\{z: k s \leq \operatorname{Re} z \leq(k+1) s, b \leq \operatorname{Im} z \leq b+s\}
$$

for all integers $k$.

Since $D$ contains $\infty$, there exists an integer $j_{0}$ such that $\partial D \subset\left\{z:|z|<j_{0}\right\}$. Hence we may choose a point $w_{-j_{0}} \in\left(\partial S_{-j_{0}}\right)_{l} \cap D$. By repeatedly applying the supposition $\left(14^{\prime}\right)$ for $k=-j_{0},-j_{0}+1, \ldots, j_{0}-1$, we find points $w_{k} \in\left(\partial S_{k}\right)_{r} \cap D=$ $\left(\partial S_{k+1}\right)_{l} \cap D$ and rectifiable arcs $\gamma_{k}$ in $S_{k}$ joining $w_{k}$ to $w_{k+1}$. It follows that $\sum_{k=-j_{0}}^{j_{0}-1} \gamma_{k}$ is a rectifiable arc in $D \cap\left(\bigcup_{k=-j_{0}}^{j_{0}-1} S_{k}\right)$ joining $w_{-j_{0}}$ to $w_{j_{0}}$. By Lemma 3.5 we may replace this arc with a Jordan $\operatorname{arc} \Gamma_{1}$ having the same properties. The points $w_{-j_{0}}$ and $w_{j_{0}}$ can clearly be connected by a Jordan $\operatorname{arc} \Gamma_{2}$ in $D \backslash\left\{z:|z|<j_{0}\right\}$. But then $\Gamma=\Gamma_{1} \cup \Gamma_{2}$ is a Jordan curve separating the points $\zeta_{1}$ and $\zeta_{2}$; this contradicts the fact that $\partial D$ is connected. Therefore the first assertion of the lemma is shown.

To show the second claim, let $s_{0}$ be given and assume that $S=\{z: a \leq \operatorname{Re} z \leq$ $a+s, b \leq \operatorname{Im} z \leq b+s\}$ is as described in (14). Choose a point $z_{1} \in(\partial S)_{l} \cap D$ such that for any point $z_{2} \in(\partial S)_{r} \cap D$, there does not exist a rectifiable arc in $D \cap S$ joining $z_{1}$ to $z_{2}$.

We now construct the disk $\Delta^{*}$. Choose $r>0$ such that

$$
\Delta_{1}=\left\{z:\left|z-z_{1}\right|<r\right\} \subset D,
$$

and choose a point $z^{*} \in \Delta_{1} \cap S$. Choose $r^{*}>0$ so small that

$$
\begin{gathered}
\Delta^{*}=\left\{z:\left|z-z^{*}\right|<r^{*}\right\} \subset \Delta_{1} \cap S \text { and } \\
2 r^{*} \leq \min _{x \in[a, a+s]}\left\{\left[\left(x-\operatorname{Re} z^{*}\right)^{2}+\operatorname{dist}\left(z^{*}, \partial S\right)^{2}\right]^{1 / 2}-\left|x-\operatorname{Re} z^{*}\right|\right\} .
\end{gathered}
$$

Define $\rho^{*}(z)$ as in (16).

Let $w_{1}, w_{2} \in \partial S$ and suppose that $\gamma$ is a rectifiable arc in $D \cap S$ joining $w_{1}$ to $w_{2}$. It may be assumed that $\gamma \cap \Delta^{*} \neq \varnothing$. By construction, any point in $\Delta^{*}$ can be joined to $z_{1}$ by a rectifiable arc in $D \cap S$, thus it follows that neither $w_{1}$ nor $w_{2}$ can belong to $(\partial S)_{r}$. Inequality (15) is trivial if both $w_{1}$ and $w_{2}$ belong to $(\partial S)_{l}$, 
hence assume that $w_{2} \notin(\partial S)_{l}$. In this case we have

$$
\begin{aligned}
\int_{\gamma} \rho^{*}(z)|d z| & \geq\left|w_{1}-z^{*}\right|-r^{*}+\left|w_{2}-z^{*}\right|-r^{*} \\
& =\left|w_{1}-z^{*}\right|+\left[\left(\operatorname{Re} w_{2}-\operatorname{Re} z^{*}\right)^{2}+\left(\operatorname{Im} w_{2}-\operatorname{Im} z^{*}\right)^{2}\right]^{1 / 2}-2 r^{*} \\
& \geq\left|\operatorname{Re} w_{1}-\operatorname{Re} z^{*}\right|+\left[\left(\operatorname{Re} w_{2}-\operatorname{Re} z^{*}\right)^{2}+\operatorname{dist}\left(z^{*}, \partial S\right)^{2}\right]^{1 / 2}-2 r^{*} \\
& \geq\left|\operatorname{Re} w_{1}-\operatorname{Re} z^{*}\right|+\left|\operatorname{Re} w_{2}-\operatorname{Re} z^{*}\right| \\
& \geq\left|\operatorname{Re} w_{1}-\operatorname{Re} w_{2}\right|
\end{aligned}
$$

which shows that (15) holds and finishes the proof of the lemma.

Theorem 3.6. Let $\Omega$ be a punctured rectangular domain of length a and height $b$, and suppose that $(\Omega, \mathcal{B}, \Gamma)$ is a crossing-module problem with $\Gamma$ joining the vertical sides of $\Omega$. If there exists $B^{*} \in \mathcal{B}(\Omega) \backslash\left(\mathcal{B} \cup\left\{\partial R_{a, b}\right\}\right)$ that is not a horizontal slit and such that $\operatorname{dist}\left(B^{*}, \mathcal{B}\right)=\inf \left\{\left|\zeta^{*}-\zeta\right|: \zeta^{*} \in B^{*}, \zeta \in(\bigcup \mathcal{B})\right\}>0$, then

$$
\mu(\Gamma)<b / a .
$$

Proof. Suppose that $B^{*} \in \mathcal{B}(\Omega) \backslash\left(\mathcal{B} \cup\left\{\partial R_{a, b}\right\}\right)$ is not a horizontal slit and that $s_{0}=$ $\frac{1}{2} \operatorname{dist}\left(B^{*}, \mathcal{B}\right)>0$. Apply Lemma 3.4 with the domain $D$ equal to the unbounded component of $B^{* c}$, to obtain a square region $S$ satisfying (14) and a disk $\Delta^{*} \subset S$ such that (15) holds where $\rho^{*}(z)$ is defined by (16). Since $\Omega \subset D, \rho^{*}(z)$ restricts to a metric on $\Omega$.

In order for (14) to hold it must be the case that $B^{*} \cap S \neq \varnothing$, thus

$$
\zeta \in S \Rightarrow \operatorname{dist}\left(\zeta, B^{*}\right)<\sqrt{2} s_{0}<\operatorname{dist}\left(B^{*}, \mathcal{B}\right)
$$

This shows that $S$ does not contain any points on any crossing-boundaries, and thus that

$$
A_{\rho^{*}}(\Omega, \mathcal{B}) \leq a b-\operatorname{area}\left(\Delta^{*}\right)
$$

We shall use inequality (15) of Lemma 3.4 to obtain a lower bound for $L_{\rho^{*}}(\Gamma)$. Let $\sigma=\sum_{j=1}^{n} \gamma_{j} \in \Gamma$ be given. Since each $\gamma_{j}$ is rectifiable we may break $\gamma_{j}$ into finitely many subarcs which alternately avoid $\Delta^{*}$ and enter $\Delta^{*}$. For each $j$, choose $m_{j} \geq 0$ and collections $\left\{\alpha_{j, k}\right\}_{k=0}^{m_{j}}$ and $\left\{\beta_{j, k}\right\}_{k=1}^{m_{j}}$ of arcs such that

$$
\gamma_{j}=\alpha_{j, 0}+\sum_{k=1}^{m_{j}}\left(\beta_{j, k}+\alpha_{j, k}\right),
$$

where for each $k$ :

(i) $\alpha_{j, k}$ is an arc in $\Omega \backslash \Delta^{*}$,

(ii) $\beta_{j, k}$ contains a point of $\Delta^{*}$,

(iii) the endpoints of $\beta_{j, k}$ belong to $\partial S$, and

(iv) the interior of $\beta_{j, k}$ is contained in $S$. 
Let $a_{j, k}$ and $b_{j, k}$ denote respectively the initial and terminal points of each $\alpha_{j, k}$, and note that $b_{j, k-1}$ and $a_{j, k}$ are the initial and terminal points of $\beta_{j, k}$. By inequality (15) and definitions, we have that

$$
\begin{aligned}
l_{\rho^{*}}(\sigma)= & \sum_{j=1}^{n} \int_{\gamma_{j}} \rho^{*}(z)|d z|+\sum_{j=1}^{n-1}\left|a_{j+1,0}-b_{j, m_{j}}\right| \\
= & \sum_{j=1}^{n}\left[\int_{\alpha_{j, 0}}|d z|+\sum_{k=1}^{m_{j}}\left(\int_{\beta_{j, k}} \rho^{*}(z)|d z|+\int_{\alpha_{j, k}}|d z|\right)\right]+\sum_{j=1}^{n-1}\left|a_{j+1,0}-b_{j, m_{j}}\right| \\
\geq & \sum_{j=1}^{n}\left[\left|\operatorname{Re} b_{j, 0}-\operatorname{Re} a_{j, 0}\right|+\sum_{k=1}^{m_{j}}\left(\left|\operatorname{Re} a_{j, k}-\operatorname{Re} b_{j, k-1}\right|+\left|\operatorname{Re} b_{j, k}-\operatorname{Re} a_{j, k}\right|\right)\right] \\
& \quad+\sum_{j=1}^{n-1}\left|\operatorname{Re} a_{j+1,0}-\operatorname{Re} b_{j, m_{j}}\right| .
\end{aligned}
$$

Hence, by repeated use of the triangle inequality, we find that $l_{\rho^{*}}(\sigma) \geq a$. Since $\sigma \in \Gamma$ was arbitrary we have that $L_{\rho^{*}}(\Gamma) \geq a$. Therefore,

$$
\frac{A_{\rho^{*}}(\Omega, \mathcal{B})}{L_{\rho^{*}}(\Gamma)^{2}} \leq \frac{a b-\operatorname{area}\left(\Delta^{*}\right)}{a^{2}}=\frac{b}{a}-\frac{\operatorname{area}\left(\Delta^{*}\right)}{a^{2}}<\frac{b}{a}
$$

which shows $\mu(\Gamma)<b / a$ and completes the proof.

\section{Existence of Rectilinear Slit Maps}

In this section the existence of rectilinear slit maps achieving continuous angle assignments with finite ranges will be shown for arbitrary domains. Additionally it will be shown that the image of the rectilinear slit maps satisfy a certain crossingmodule condition called $\left(\mathrm{NP}_{\Theta}\right)$ (see Theorem 4.4) which generalizes Jenkins' $\left(\mathrm{P}_{\theta}\right)$ condition. The combination of Theorem 4.4 with the uniqueness result of Maitani and Minda [MM] provides a complete generalization of Grötzsch and Jenkins' Theorem (Theorem 1.1) for the case of rectilinear slit maps achieving continuous angle assignments with finite ranges.

In order to facilitate the statement and proof of Theorem 4.4 we introduce some notation and give some approximation lemmas.

Definition. If $\Omega$ is a domain containing $\infty$ and $\gamma$ is a Jordan curve in $\Omega$ then the open subset $\{C \in \mathcal{C}(\Omega): C \subset \operatorname{In}[\gamma]\}$ of $\mathcal{C}(\Omega)$ shall be denoted by $\mathcal{N}(\gamma)$.

Proofs of the following lemmas may be found in [W].

Lemma 4.1. Let $\Omega$ be a domain containing $\infty$. If $\left\{\mathcal{C}_{k}\right\}_{k=1}^{n}$ is a collection of disjoint closed subsets of $\mathcal{C}(\Omega)$ which partition $\mathcal{C}(\Omega)$, then there exists disjoint Jordan curves $\gamma_{1}, \gamma_{2}, \ldots, \gamma_{n}$ in $\Omega$ such that $\mathcal{C}_{k}=\mathcal{N}\left(\gamma_{k}\right)$ for $k=1,2, \ldots, n$.

Lemma 4.2. Let $\Omega$ be an $n$-connected domain containing $\infty$ with boundary components $B_{1}, B_{2}, \ldots, B_{n}$. If $\epsilon>0$, then

(i) There exists disjoint piecewise linear Jordan curves $J_{1}, J_{2}, \ldots, J_{n}$ in $\Omega$ such that for $l=1,2, \ldots, n$,

$$
\begin{gathered}
B_{k} \subset \operatorname{In}\left[J_{l}\right] \quad \text { if and only if } k=l \text {, and } \\
z \in \Omega \cap \operatorname{In}\left[J_{l}\right] \quad \Rightarrow \quad \operatorname{dist}\left(z, J_{l}\right)<\epsilon .
\end{gathered}
$$


(ii) Moreover, if $B_{1}, B_{2}, \ldots, B_{n}$ are known to be rectifiable Jordan curves, then $J_{1}, J_{2}, \ldots, J_{n}$ can be chosen to additionally satisfy

$$
\sum_{k=1}^{n} \operatorname{area}\left(\operatorname{In}\left[J_{k}\right] \backslash \operatorname{In}\left[B_{k}\right]\right)<\epsilon^{\prime}
$$

for any $\epsilon^{\prime}>0$.

Lemma 4.3. Suppose that $\Omega$ is a bounded domain such that the unbounded complementary component, $C_{\infty}$, is an isolated component. Then there exists a piecewise linear Jordan curve $J$ in $\Omega$ which encloses every complementary component of $\Omega$ except for $C_{\infty}$.

The condition $\left(\mathrm{NP}_{\Theta}\right)$ involves a collection of crossing-module problems. To describe such a collection, we first show how a continuous angle assignment with finite range can induce a set of crossing-boundaries on a domain.

Consider a domain $D$ containing $\infty$, and suppose that $\Phi$ is a continuous angle assignment on $\mathcal{C}(D)$ which has range $\left\{\phi_{1}, \phi_{2}, \ldots, \phi_{n}\right\}$. For each $j=1,2, \ldots, n$,

$$
\mathcal{C}_{j}=\Phi^{-1}\left(\phi_{j}\right)
$$

is a closed subset of $\mathcal{C}(D)$, and the collection $\left\{\mathcal{C}_{j}\right\}_{j=1}^{n}$ forms a partition of $\mathcal{C}(D)$. Hence Lemmas 4.1 and 4.2 imply the existence of disjoint, rectifiable Jordan curves $B_{1}, B_{2}, \ldots, B_{n}$ in $D$ satisfying

$$
\mathcal{C}_{j}=\mathcal{N}\left(B_{j}\right) \quad \text { for } j=1,2, \ldots, n .
$$

Definition. If $D$ is a domain containing $\infty$ and $\Phi$ is a continuous angle assignment on $D$ with range $\left\{\phi_{1}, \phi_{2}, \ldots, \phi_{n}\right\}$ then, any collection $\mathcal{B}=\left\{B_{1}, B_{2}, \ldots, B_{n}\right\}$ of disjoint rectifiable Jordan curves in $D$ satisfying (18) where $\mathcal{C}_{j}$ is defined by (17) is said to be a $\Phi$-induced crossing-boundary collection in $D$.

Theorem 4.4. Suppose that $\Omega$ is a domain containing $\infty$, and that $\Theta$ is a continuous angle assignment of finite range $\left\{\theta_{1}, \theta_{2}, \ldots, \theta_{n}\right\}$. Then there exists a map $f(z)$ achieving $\Theta$ with the expansion

$$
f(z)=z+\frac{a_{1}}{z}+\cdots \quad \text { near } \infty
$$

that satisfies the following condition which will be referred to as $\left(N P_{\Theta}\right)$.

If $\mathcal{B}=\left\{B_{1}, B_{2}, \ldots, B_{n}\right\}$ is any $\Theta \circ\left(f^{-1}\right)^{\mathcal{C}}$-induced crossing-boundary collection in $f(\Omega)$, and $s$ is sufficiently large so that

$$
\left(f(\Omega)^{c} \cup(\bigcup \mathcal{B})\right) \subset S_{s, \phi} \quad \text { for all } \phi \in\left\{\theta_{1}, \theta_{2}, \ldots, \theta_{n}\right\}
$$

where $S_{s, \phi}=\left\{e^{i \phi} z:|\operatorname{Re} z|<s,|\operatorname{Im} z|<s\right\}$, then for each $j=1,2, \ldots, n$, the crossing-module problem $\left(D_{j}, \mathcal{B}_{j}, \Gamma_{j}\right)$ defined by

$$
\left\{\begin{array}{l}
D_{j}=\left(f(\Omega) \cap S_{s, \theta_{j}}\right) \backslash \bigcup_{k \neq j} \overline{\operatorname{In}\left[B_{k}\right]} \\
\mathcal{B}_{j}=\mathcal{B} \backslash\left\{B_{j}\right\}, \text { and } \\
\Gamma_{j}=\left\{\sigma \in C_{D_{j}}\left(\mathcal{B}_{j}\right): \sigma \text { joins the sides of } S_{s, \theta_{j}} \text { at inclination } \theta_{j}+\pi / 2\right\},
\end{array}\right.
$$

has crossing-module

$$
\mu\left(\Gamma_{j}\right)=1
$$

(See Figure 4 for an illustration of such a crossing-module problem.) 


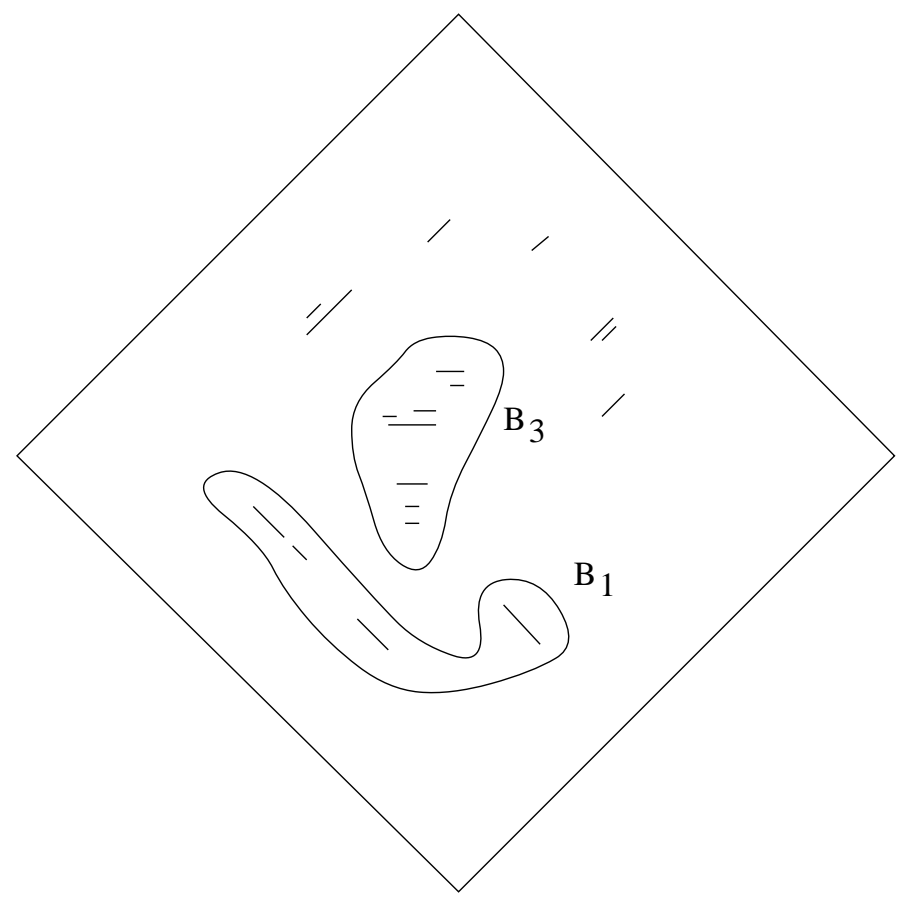

FiguRE 4. Illustration of a crossing-module problem $\left(D_{2}, \mathcal{B}_{2}, \Gamma_{2}\right)$.

Proof. It suffices to assume that $n \geq 2$, for in the case of $n=1$ (i.e., a parallel assignment) the crossing-module may be replaced with the module and then the statement of the theorem coincides with part of an established theorem (Theorem 1.1) proven in [J, pp. 81-84] (see specifically Lemma 5.8 on p. 82).

We first construct a candidate map $f(z)$. Since $\Theta$ is a continuous angle assignment with finite range, Lemmas 4.1 and 4.2 imply the existence of disjoint Jordan curves $J_{1}, J_{2}, \ldots, J_{n}$ in $\Omega$ such that $\Theta^{-1}\left(\theta_{k}\right)=\mathcal{N}\left(J_{k}\right)$ for $k=1,2, \ldots, n$. Since $\widehat{\mathbb{C}} \backslash \bigcup_{k=1}^{n} \operatorname{In}\left[J_{k}\right]$ is a compact subset of $\Omega$, we may choose an exhaustion $\left\{\Omega_{m}\right\}$ such that

$$
\widehat{\mathbb{C}} \backslash \bigcup_{k=1}^{n} \operatorname{In}\left[J_{k}\right] \subset \Omega_{m}
$$

for all $m$.

Now fix $m$ and consider a component $C$ of $\Omega_{m}^{c}$. The inclusion (21) shows that $C$ is enclosed by precisely one of the curves $J_{1}, J_{2}, \ldots, J_{n}$. Thus we may define an angle assignment $\Theta_{m}$ on $\mathcal{C}\left(\Omega_{m}\right)$ by requiring that for each $C \in \mathcal{C}\left(\Omega_{m}\right)$,

$$
\Theta_{m}(C)=\theta_{k}
$$

where $k$ is the unique integer such that $C \subset \operatorname{In}\left[J_{k}\right]$. Since $\Omega_{m}$ is finitely connected, Koebe's Theorem (Theorem 1.2) gives the existence of a map $f_{m}(z)$ achieving the angle assignment $\Theta_{m}$ and having expansion

$$
f_{m}(z)=z+\frac{a_{1, m}}{z}+\cdots \quad \text { near } \infty .
$$




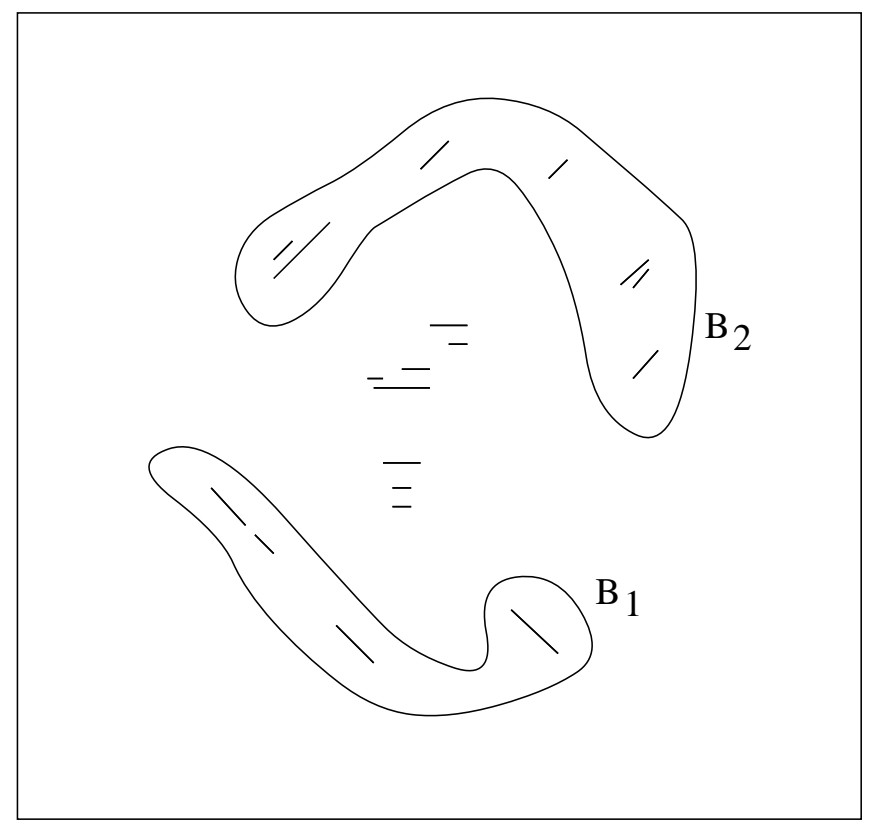

Figure 5. A crossing-module problem $\left(D_{n}, \mathcal{B}_{n}, \Gamma_{n}\right)$.

It is easy to show (see [W, p. 67]) that $\left\{f_{m}(z)\right\}$ forms a normal family and has a subsequential limit $f(z)$ that is univalent on $\Omega$ with expansion

$$
f(z)=z+\frac{a_{1}}{z}+\cdots \quad \text { near } \infty .
$$

By passing to the subsequence, it may be assumed that the entire sequence $\left\{f_{m}(z)\right\}$ converges to $f(z)$.

Now we verify that $\left(\mathrm{NP}_{\Theta}\right)$ is satisfied for this $f(z)$. Let $\mathcal{B}=\left\{B_{1}, B_{2}, \ldots, B_{n}\right\}$ be any $\Theta \circ\left(f^{-1}\right)^{\mathcal{C}}$-induced crossing-boundary collection in $f(\Omega)$, and let $s$ be sufficiently large so that (19) holds. Consider the crossing-module problem $\left(D_{j}, \mathcal{B}_{j}, \Gamma_{j}\right)$ defined by (20) for some fixed $j \in\{1,2, \ldots, n\}$. For simplicity of notation it may be assumed that $j=n$; further, since the crossing-module is invariant under rotation we may assume that $\theta_{n}=0$ (see the illustration in Figure 5 ). We shall also use the notation $S_{s}=S_{s, 0}$, and denote the left and right sides of the square $\partial S_{s}$ by $\left(\partial S_{s}\right)_{l}$ and $\left(\partial S_{s}\right)_{r}$ respectively.

For the metric $\rho^{*}(z) \equiv 1$ on $D_{n}$ it is clear that $A_{\rho^{*}}\left(D_{n}, \mathcal{B}_{n}\right) \leq s^{2}$ and that $L_{\rho^{*}}\left(\Gamma_{n}\right) \geq s$, hence the inequality

$$
\mu\left(\Gamma_{n}\right) \leq 1
$$

is immediate. Showing the reverse inequality shall require much more work. A succession of five crossing-module problems will be constructed. The value of crossingmodule of the final problem will be found by using the theorem giving the crossingmodule of a horizontally sectionable, punctured rectangular domain (Theorem 3.3). While the necessary chain of inequalities relating the crossing-module problems will follow from various estimates and the theorem on the conformal variance of the crossing-module (Theorem 3.1). 
In order to apply Theorem 3.1 the crossing-number of all chains in the crossingmodule problem under consideration must be uniformly bounded. For each positive integer $N$, let

$$
\Gamma_{n}^{N}=\left\{\sigma \in \Gamma_{n}: \mathrm{c}_{\#}(\sigma) \leq N\right\} .
$$

The inclusion $\Gamma_{n}^{N} \subset \Gamma_{n}$ implies that $L_{\rho}\left(\Gamma_{n}^{N}\right) \geq L_{\rho}\left(\Gamma_{n}\right)$ for any metric $\rho(z)$ on $D_{n}$, and hence that

$$
\mu\left(\Gamma_{n}\right) \geq \mu\left(\Gamma_{n}^{N}\right) .
$$

Therefore, in order to prove that $\mu\left(\Gamma_{n}\right) \geq 1$, it suffices to show that for each $\delta>0$ there exists $N$ such that

$$
\mu\left(\Gamma_{n}^{N}\right) \geq 1-\delta
$$

Let $\delta>0$ be given. The choice of $N$ will be determined by approximating the curves $B_{k}$ from the inside and outside by piecewise linear curves. The inner curves shall serve as the crossing-boundaries in a yet to be specified crossing-module problem. By choosing $N$ sufficiently large this problem will be assured to be horizontally sectionable. For each $k=1,2, \ldots, n$, apply Lemma 4.3 to the domain $\operatorname{In}\left[B_{k}\right] \cap f(\Omega)$ to obtain a piecewise linear Jordan curve $I_{k}$ in $f(\Omega)$ that satisfies

$$
I_{k} \subset \operatorname{In}\left[B_{k}\right] \quad \text { and } \quad \mathcal{N}\left(I_{k}\right)=\mathcal{N}\left(B_{k}\right) .
$$

Next apply Lemma 4.2 to the domain $f(\Omega) \backslash \bigcup_{k=1}^{n} \operatorname{In}\left[B_{k}\right]$ and $\epsilon=\operatorname{dist}\left(\bigcup \mathcal{B}, S_{s}\right)$, to obtain a collection $\left\{O_{k}\right\}_{k=1}^{n}$ of disjoint piecewise linear Jordan curves in $f(\Omega) \backslash$ $\bigcup_{k=1}^{n} \operatorname{In}\left[B_{k}\right]$ each contained in $S_{s}$ that satisfies

$$
B_{k} \subset \operatorname{In}\left[O_{j}\right] \quad \text { if and only if } j=k,
$$

for $k=1,2, \ldots, n$, and also the condition

$$
\sum_{k=1}^{n} \operatorname{area}\left(\operatorname{In}\left[O_{k}\right] \backslash \operatorname{In}\left[B_{k}\right]\right)<\frac{\delta}{4} A_{b}\left(\mathcal{B}_{n}\right) .
$$

This construction is illustrated in Figure 6. Define $N$ to be the total number of segments comprising all of the curves $O_{k}$ for $k=1,2, \ldots, n$.

We shall show the crossing-module problem $\left(D_{n}, \mathcal{B}_{n}, \Gamma_{n}^{N}\right)$ has crossing-module greater than or equal to $1-\delta$. Since $n \geq 2$ the area bounded by the crossingboundaries $A_{b}\left(\mathcal{B}_{n}\right)$ is strictly positive. This observation allows one to limit the class of metrics for which analysis must be performed; in particular we note that for any metric $\rho(z)$ on $D_{n}$,

$$
L_{\rho}\left(\Gamma_{n}^{N}\right) \leq A_{b}\left(\mathcal{B}_{n}\right)^{1 / 2} \Rightarrow \frac{A_{\rho}\left(D_{n}, \mathcal{B}_{n}\right)}{L_{\rho}\left(\Gamma_{n}^{N}\right)^{2}} \geq \frac{A_{b}\left(\mathcal{B}_{n}\right)}{A_{b}\left(\mathcal{B}_{n}\right)}=1 .
$$

Thus it suffices to consider a metric $\rho(z)$ on $D_{n}$ such that $L_{\rho}\left(\Gamma_{n}^{N}\right)>A_{b}\left(\mathcal{B}_{n}\right)^{1 / 2}$. Let $\rho(z)$ be such a metric and let $\sigma^{N}$ be a chain in $\Gamma_{n}^{N}$ such that $l_{\rho}\left(\sigma^{N}\right) \leq 2 L_{\rho}\left(\Gamma_{n}^{N}\right)$.

Since $\left\{\Omega_{m}\right\}$ exhausts $\Omega$ and $f(z)$ is a homeomorphism onto $f(\Omega)$, the sequence of domains $\left\{f\left(\Omega_{m}\right)\right\}$ exhausts $f(\Omega)$. Hence a compactness argument shows that there exists $M$ such that $\sigma^{N}$ and the domain $\widehat{\mathbb{C}} \backslash \bigcup_{k=1}^{n} \operatorname{In}\left[I_{k}\right]$ are contained in $f\left(\Omega_{m}\right)$ for each $m \geq M$.

Consider, for each $m=1,2, \ldots$, the map $g_{m}: f\left(\Omega_{m}\right) \rightarrow f_{m}\left(\Omega_{m}\right)$ defined by

$$
g_{m}(z)=f_{m} \circ f^{-1}(z) .
$$




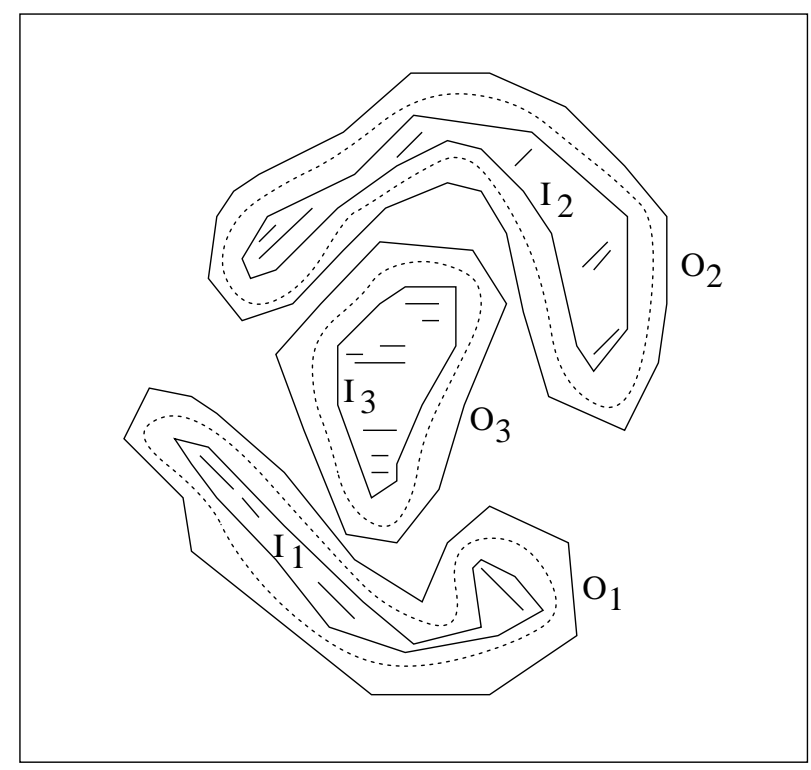

Figure 6 . The curves $O_{k}$ and $I_{k}$.

Since $\left\{f_{m}(z)\right\}$ converges normally to $f(z)$, the sequence $\left\{g_{m}(z)\right\}$ converges normally to $g(z)=z$ on $f(\Omega)$. Let $\epsilon>0$ be less than $\min \left\{1, \epsilon_{0}, \epsilon_{1}, \epsilon_{2}, \epsilon_{3}, \epsilon_{4}\right\}$, where

$$
\begin{gathered}
\epsilon_{0}=A_{b}\left(\mathcal{B}_{n}\right)^{1 / 2} /(4 N), \quad \epsilon_{1}=\min \operatorname{arclength}\left(B_{k}\right) \\
\epsilon_{2}=\min \operatorname{dist}\left(I_{k}, B_{k}\right), \quad \epsilon_{3}=\min \operatorname{dist}\left(B_{k}, O_{k}\right), \quad \epsilon_{4}=\min \operatorname{dist}\left(O_{k}, \partial S_{s}\right)
\end{gathered}
$$

and the minimums are taken over $k=1,2, \ldots, n$. Choose $m_{0} \geq M$ such that

$$
\left|g_{m_{0}}(z)-z\right|<\epsilon \quad \text { on } \quad \widehat{\mathbb{C}} \backslash \bigcup_{k=1}^{n} \operatorname{In}\left[I_{k}\right] .
$$

By choice of $M$, each of the crossing-boundaries in $\mathcal{B}_{n}$ is a boundary component of $D_{n} \cap f\left(\Omega_{m_{0}}\right)$. Thus we may consider the crossing-module problem $\left(D_{n}^{0}, \mathcal{B}_{n}^{0}, \Gamma_{n}^{0}\right)$, illustrated in Figure 7, where the domain $f(\Omega)$ is replaced by $f\left(\Omega_{m}\right)$; that is where

$$
\begin{aligned}
& D_{n}^{0}=D_{n} \cap f\left(\Omega_{m_{0}}\right), \\
& \mathcal{B}_{n}^{0}=\mathcal{B}_{n}, \quad \text { and } \\
& \Gamma_{n}^{0}=\left\{\sigma \in \Gamma_{n}^{N}: \sigma \in C_{D_{n}^{0}}\left(\mathcal{B}_{n}^{0}\right)\right\} .
\end{aligned}
$$

Let $\rho_{0}$ be the metric on $D_{n}^{0}$ defined by restricting $\rho(z)$,

$$
\rho_{0}(z)=\left.\rho(z)\right|_{D_{n}^{0}} .
$$

Since $D_{n}^{0} \subset D_{n}$ and $\mathcal{B}_{n}^{0}=\mathcal{B}_{n}$ it is clear that $A_{\rho_{0}}\left(D_{n}^{0}, \mathcal{B}_{n}^{0}\right) \leq A_{\rho}\left(D_{n}, \mathcal{B}_{n}\right)$, while the inclusion $\Gamma_{n}^{0} \subset \Gamma_{n}^{N}$ implies that $L_{\rho_{0}}\left(\Gamma_{n}^{0}\right) \geq L_{\rho}\left(\Gamma_{n}^{N}\right)$. Hence

$$
\frac{A_{\rho}\left(D_{n}, \mathcal{B}_{n}\right)}{L_{\rho}\left(\Gamma_{n}^{N}\right)^{2}} \geq \frac{A_{\rho_{0}}\left(D_{n}^{0}, \mathcal{B}_{n}^{0}\right)}{L_{\rho_{0}}\left(\Gamma_{n}^{0}\right)^{2}}
$$

Also, since $m_{0} \geq M$, the chain $\sigma^{N}$ belongs to $\Gamma_{n}^{0}$ which implies that

$$
L_{\rho_{0}}\left(\Gamma_{n}^{0}\right) \leq 2 L_{\rho}\left(\Gamma_{n}^{N}\right) \text {. }
$$




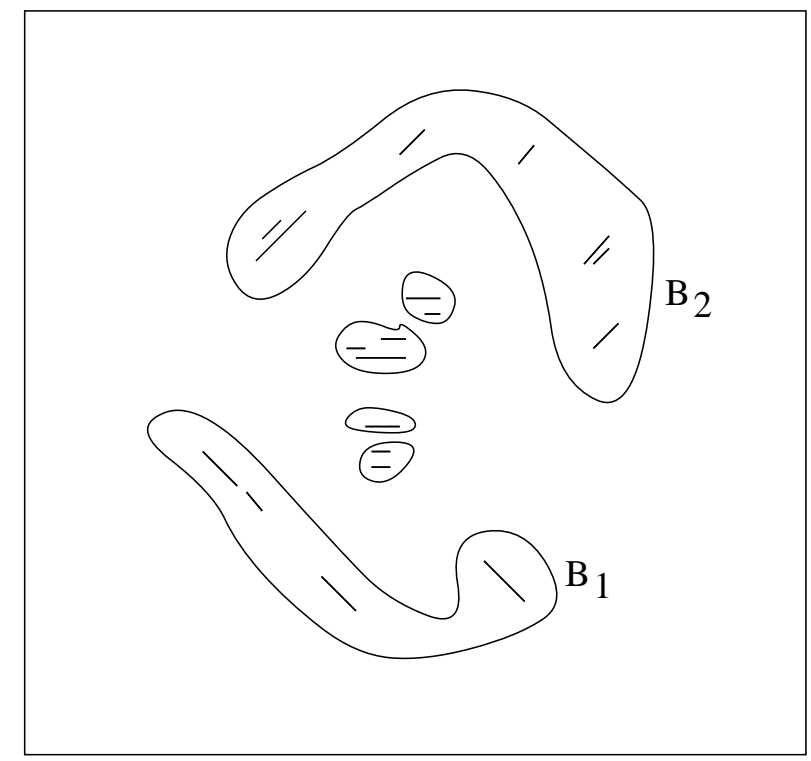

Figure 7. A crossing-module problem $\left(D_{n}^{0}, \mathcal{B}_{n}^{0}, \Gamma_{n}^{0}\right)$.

Consider next the crossing-module problem $\left(D_{n}^{1}, \mathcal{B}_{n}^{1}, \Gamma_{n}^{1}\right)$ induced by the mapping $g_{m_{0}}(z)$ on the crossing-module problem $\left(D_{n}^{0}, \mathcal{B}_{n}^{0}, \Gamma_{n}^{0}\right)$, that is where

$$
\begin{aligned}
D_{n}^{1} & =g_{m_{0}}\left(D_{n}^{0}\right), \\
\mathcal{B}_{n}^{1} & =\left\{g_{m_{0}}\left(B_{1}\right), g_{m_{0}}\left(B_{2}\right), \ldots, g_{m_{0}}\left(B_{n-1}\right)\right\}, \text { and } \\
\Gamma_{n}^{1} & =\left\{g_{m_{0}}(\sigma): \sigma \in \Gamma_{n}^{0}\right\} .
\end{aligned}
$$

This problem is illustrated in Figure 8. Since $g_{m_{0}}(z)$ is a homeomorphism of $D_{n}^{0}$ onto $D_{n}^{1}$, the set $\Gamma_{n}^{1}$ consists of all chains $\sigma \in C_{D_{n}^{1}}\left(\mathcal{B}_{n}^{1}\right)$ joining $g_{m_{0}}\left(\left(\partial S_{s}\right)_{l}\right)$ to $g_{m_{0}}\left(\left(\partial S_{s}\right)_{r}\right)$ whose crossing-numbers are bounded by $N$.

Let $\rho_{1}(z)=\rho_{0}{ }^{g_{m_{0}}}(z)$ be the induced metric on $D_{n}^{1}$ defined as in equation (6). We shall use Theorem 3.1 to obtain a lower bound for $A_{\rho_{0}}\left(D_{n}^{0}, \mathcal{B}_{n}^{0}\right) / L_{\rho_{0}}\left(\Gamma_{n}^{0}\right)^{2}$ in terms of $A_{\rho_{1}}\left(D_{n}^{1}, \mathcal{B}_{n}^{1}\right) / L_{\rho_{1}}\left(\Gamma_{n}^{1}\right)^{2}$. The choice of $m_{0}$ implies that

$$
|| g_{m_{0}}\left(z_{1}\right)-g_{m_{0}}\left(z_{2}\right)|-| z_{1}-z_{2}||<2 \epsilon
$$

for $z_{1}, z_{2} \in B_{k}$, and $k=1,2, \ldots, n$. Hence inequality (8) implies that

$$
L_{\rho_{1}}\left(\Gamma_{n}^{1}\right) \geq L_{\rho_{0}}\left(\Gamma_{n}^{0}\right)-2 \epsilon N .
$$

Since $\epsilon<\epsilon_{0}=A_{b}\left(\mathcal{B}_{n}\right)^{1 / 2} /(4 N)$ and $L_{\rho_{0}}\left(\Gamma_{n}^{0}\right) \geq L_{\rho}\left(\Gamma_{n}^{N}\right)$, this inequality is nontrivial; indeed,

$$
L_{\rho_{0}}\left(\Gamma_{n}^{0}\right)-2 \epsilon N>\frac{1}{2} A_{b}\left(\mathcal{B}_{n}\right)^{1 / 2} .
$$

Also observe that $\epsilon<\epsilon_{1}$ and Lemma 3.2 imply that

$$
\left|A_{b}\left(\mathcal{B}_{n}^{1}\right)-A_{b}\left(\mathcal{B}_{n}^{0}\right)\right|<\kappa_{1} \epsilon
$$




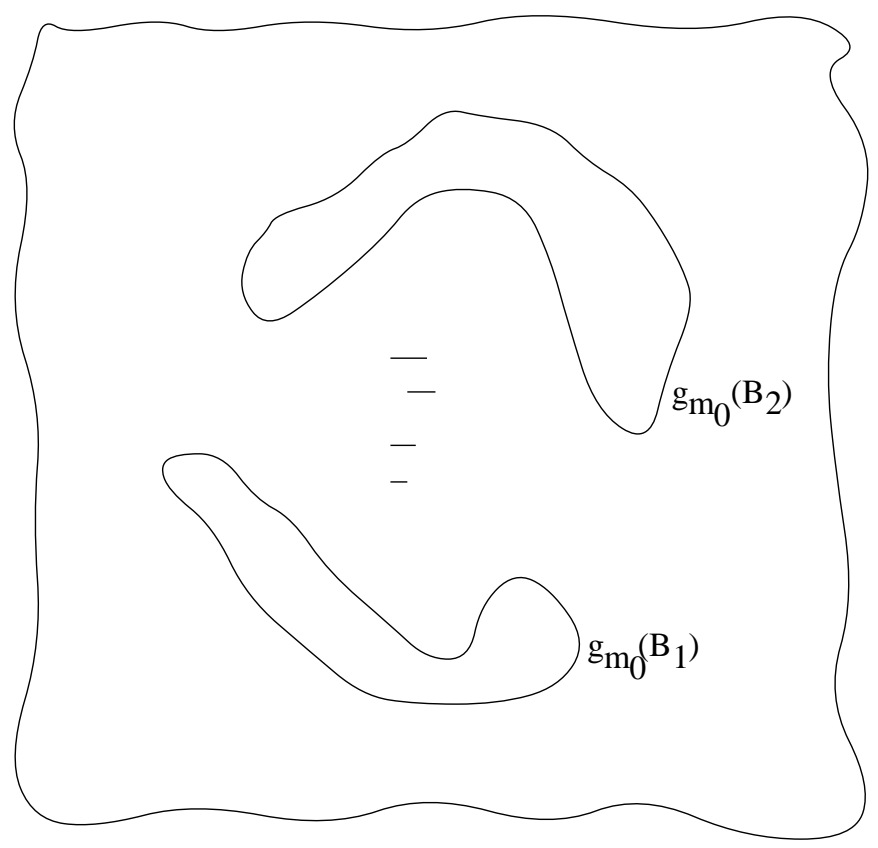

Figure 8. A crossing-module problem $\left(D_{n}^{1}, \mathcal{B}_{n}^{1}, \Gamma_{n}^{1}\right)$.

where $\kappa_{1}=\sum_{k=1}^{n} \operatorname{arclength}\left(B_{k}\right) /(8 \pi)$ is a constant independent of $\epsilon$. Now estimate (10), the fact that $\epsilon<1$, and inequalities (24) through (29) yield that

$$
\frac{A_{\rho_{0}}\left(D_{n}^{0}, \mathcal{B}_{n}^{0}\right)}{L_{\rho_{0}}\left(\Gamma_{n}^{0}\right)^{2}} \geq \frac{A_{\rho_{1}}\left(D_{n}^{1}, \mathcal{B}_{n}^{1}\right)}{L_{\rho_{1}}\left(\Gamma_{n}^{1}\right)^{2}}-\frac{4}{A_{b}\left(\mathcal{B}_{n}\right)}\left[\kappa_{1}+2 N\left(4 L_{\rho}\left(\Gamma_{n}^{N}\right)+2 N\right) \frac{A_{\rho}\left(D_{n}, \mathcal{B}_{n}\right)}{L_{\rho}\left(\Gamma_{n}^{N}\right)^{2}}\right] \epsilon .
$$

Hence

$$
\frac{A_{\rho_{0}}\left(D_{n}^{0}, \mathcal{B}_{n}^{0}\right)}{L_{\rho_{0}}\left(\Gamma_{n}^{0}\right)^{2}} \geq \frac{A_{\rho_{1}}\left(D_{n}^{1}, \mathcal{B}_{n}^{1}\right)}{L_{\rho_{1}}\left(\Gamma_{n}^{1}\right)^{2}}-\kappa_{2} \epsilon
$$

where $\kappa_{2}>0$ is a constant independent of $\epsilon$.

We now examine the geometry of $D_{n}^{1}$. The outer boundary component $g_{m_{0}}\left(\partial S_{s}\right)$ is a Jordan curve which is close to the square $\partial S_{s}$ in the sense that

$$
S_{s-\epsilon} \subset \operatorname{In}\left[g_{m_{0}}\left(\partial S_{s}\right)\right] \subset S_{s+\epsilon} .
$$

Further note that $g_{m_{0}}\left(\partial S_{s}\right)$ encloses all of the curves $O_{k}$, since $\epsilon<\epsilon_{4}$. Also, since $\epsilon$ is less than $\epsilon_{2}$ and $\epsilon_{3}$, each of the crossing-boundaries $g_{m_{0}}\left(B_{k}\right) \in \mathcal{B}_{n}^{1}$ is a perturbed version of $B_{k}$ which lies in the region between the curves $I_{k}$ and $O_{k}$. In other words, we have the inclusions

$$
I_{k} \subset \operatorname{In}\left[g_{m_{0}}\left(B_{k}\right)\right] \subset \operatorname{In}\left[O_{k}\right]
$$

for $k=1,2, \ldots, n-1$. Finally, it follows from the definition of $f_{m_{0}}(z)$, that all the remaining boundary components of $D_{n}^{1}$ are horizontal slits enclosed by $O_{n}$ and that there are only finitely many of these slits.

Consider next the crossing-module problem $\left(D_{n}^{2}, \mathcal{B}_{n}^{2}, \Gamma_{n}^{2}\right)$, illustrated in Figure 9, obtained by replacing the outer boundary component $g_{m_{0}}\left(\partial S_{s}\right)$ by a (nearly square) 


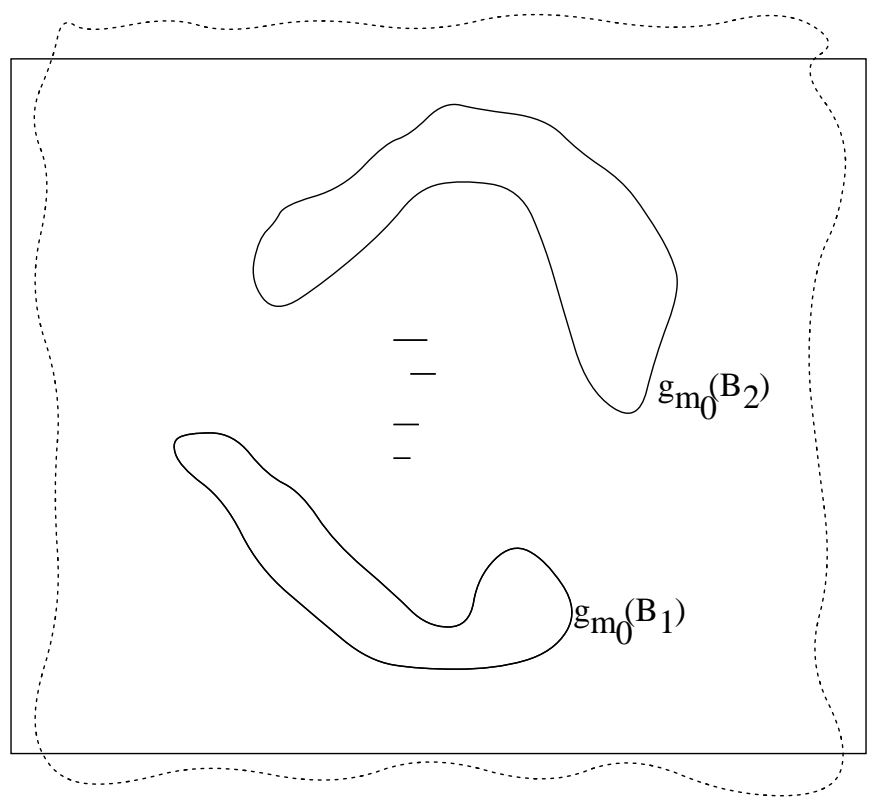

Figure 9. A crossing-module problem $\left(D_{n}^{2}, \mathcal{B}_{n}^{2}, \Gamma_{n}^{2}\right)$.

rectangle. Define

$$
\begin{aligned}
D_{n}^{2} & =\left(D_{n}^{1} \cup S_{s-\epsilon}^{c}\right) \cap R_{s+\epsilon, s-\epsilon}, \\
\mathcal{B}_{n}^{2} & =\mathcal{B}_{n}^{1}, \text { and } \\
\Gamma_{n}^{2} & =\left\{\sigma \in C_{D_{n}^{2}}\left(\mathcal{B}_{n}^{2}\right): \mathrm{c}_{\#}(\sigma) \leq N, \sigma \text { joins the vertical sides of } \partial R_{s+\epsilon, s-\epsilon}\right\},
\end{aligned}
$$

where $R_{s+\epsilon, s-\epsilon}$ is the rectangular domain

$$
R_{s+\epsilon, s-\epsilon}=\{z:|\operatorname{Re} z|<s+\epsilon, \quad|\operatorname{Im} z|<s-\epsilon\} .
$$

Let $\rho_{2}(z)$ be the metric on $D_{n}^{2}$ defined by

$$
\rho_{2}(z)= \begin{cases}\rho_{1}(z), & z \in D_{n}^{1}, \\ 0, & z \in D_{n}^{2} \backslash D_{n}^{1} .\end{cases}
$$

It is clear that $A_{\rho_{2}}\left(D_{n}^{2}, \mathcal{B}_{n}^{2}\right) \leq A_{\rho_{1}}\left(D_{n}^{1}, \mathcal{B}_{n}^{1}\right)$. On the other hand, for each $\sigma^{2} \in \Gamma_{n}^{2}$ there exists a $\sigma^{1} \in \Gamma_{n}^{1}$ contained in $\sigma^{2} \cap D_{n}^{1}$. Since $\rho_{1}(z)=\rho_{2}(z)$ on $\sigma^{1}$, the infimum of $l_{\rho_{1}}(\sigma)$ over $\sigma \in \Gamma_{n}^{1}$ is less than that of $l_{\rho_{2}}(\sigma)$ over $\sigma \in \Gamma_{n}^{2}$. Thus

$$
L_{\rho_{2}}\left(\Gamma_{n}^{2}\right) \geq L_{\rho_{1}}\left(\Gamma_{n}^{1}\right)
$$

and therefore

$$
\frac{A_{\rho_{1}}\left(D_{n}^{1}, \mathcal{B}_{n}^{1}\right)}{L_{\rho_{1}}\left(\Gamma_{n}^{1}\right)^{2}} \geq \frac{A_{\rho_{2}}\left(D_{n}^{2}, \mathcal{B}_{n}^{2}\right)}{L_{\rho_{2}}\left(\Gamma_{n}^{2}\right)^{2}} .
$$

One final modification of the crossing-module problem must be made before the area to length-squared ratio can be estimated by Theorem 3.3. The need for this last modification is due to the fact that the problem $\left(D_{n}^{2}, \mathcal{B}_{n}^{2}, \Gamma_{n}^{2}\right)$ might not be horizontally sectionable since $\Gamma_{n}^{2}$ only contains chains whose crossing-numbers are less or equal to $N$ while the crossing-boundaries $g_{m_{0}}\left(B_{k}\right)$ may oscillate up and down 


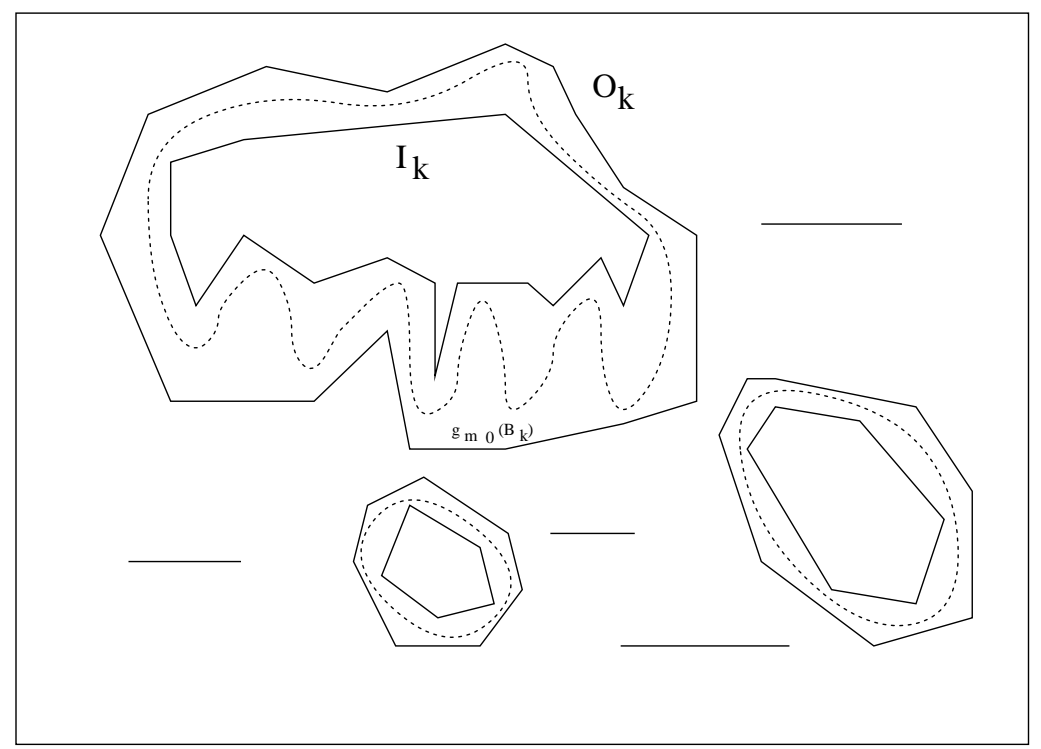

FiguRE 10. Illustration of the possible oscillation of the curves $g_{m_{0}}\left(B_{k}\right)$.

frequently (see Figure 10). To avoid this possibility we shall enlarge the domain $D_{n}^{2}$ by expanding up to the boundary curves $I_{k}(k=1,2, \ldots, n-1)$. In the new crossing-module problem it shall be sufficient to only consider the horizontal chains. Let $\left(D_{n}^{3}, \mathcal{B}_{n}^{3}, \Gamma_{n}^{3}\right)$ be the crossing-module problem defined by

$$
\begin{aligned}
D_{n}^{3} & =D_{n}^{2} \cup\left(\bigcup_{k=1}^{n-1} \overline{\operatorname{In}\left[g_{m_{0}}\left(B_{k}\right)\right]} \backslash \operatorname{In}\left[I_{k}\right]\right), \\
B_{n}^{3} & =\left\{I_{1}, I_{2}, \ldots, I_{n-1}\right\}, \text { and } \\
\Gamma_{n}^{3} & =\left\{\sigma_{y} \in C_{D_{n}^{3}}\left(\mathcal{B}_{n}^{3}\right): \sigma_{y} \text { is a horizontal chain joining the vertical sides of } D_{n}^{3}\right\} .
\end{aligned}
$$

Consider the metric $\rho_{3}(z)$ defined on $D_{n}^{3}$ by

$$
\rho_{3}(z)= \begin{cases}\rho_{2}(z), & z \in D_{n}^{3} \backslash \widehat{O} \\ \max \left\{\rho_{2}(z), 1\right\}, & z \in \widehat{O} \backslash \widehat{B^{g}} \\ 1, & z \in \widehat{B^{g}} \backslash \widehat{I}\end{cases}
$$

where

$$
\widehat{O}=\bigcup_{k=1}^{n-1} \operatorname{In}\left[O_{k}\right], \quad \widehat{B^{g}}=\bigcup_{k=1}^{n-1} \operatorname{In}\left[g_{m_{0}}\left(B_{k}\right)\right], \text { and } \quad \widehat{I}=\bigcup_{k=1}^{n-1} \operatorname{In}\left[I_{k}\right] .
$$

It follows from the definition of $\rho_{3}(z)$ and $\rho$-area that

$$
A_{\rho_{3}}\left(D_{n}^{3}, \mathcal{B}_{n}^{3}\right) \leq A_{\rho_{2}}\left(D_{n}^{2}, \mathcal{B}_{n}^{2}\right)+\operatorname{area}\left(\widehat{O} \backslash \widehat{B^{g}}\right) .
$$

The term area $\left(\widehat{O} \backslash \widehat{B^{g}}\right)$ is small; indeed the inclusions (31), the condition (22), and the inequality (29), show that area $\left(\widehat{O} \backslash \widehat{B^{g}}\right) \leq \frac{\delta}{4} A_{b}\left(\mathcal{B}_{n}\right)+\kappa_{1} \epsilon$. Therefore

$$
A_{\rho_{2}}\left(D_{n}^{2}, \mathcal{B}_{n}^{2}\right) \geq A_{\rho_{3}}\left(D_{n}^{3}, \mathcal{B}_{n}^{3}\right)-\frac{\delta}{4} A_{b}\left(\mathcal{B}_{n}\right)-\kappa_{1} \epsilon .
$$


We shall now estimate $L_{\rho_{2}}\left(\Gamma_{n}^{2}\right)$ in terms of $L_{\rho_{3}}\left(\Gamma_{n}^{3}\right)$. It is useful to keep Figure 10 in mind during the construction involved in the estimate. Let the horizontal chain $\sigma^{3} \in \Gamma_{n}^{3}$ be given. Consider the horizontal chain $\sigma^{4}$ with the same imaginary part as $\sigma^{3}$, but whose image lies in $D_{n}^{3} \backslash \widehat{O}$. In other words, $\sigma^{4}$ is a subchain of $\sigma^{3}$ which crosses the curves $O_{k}$. Since $N$ is greater than the total number of segments comprising the piecewise linear curves $O_{k}$ for $k=1,2, \ldots, n-1$, the crossing-number of $\sigma^{4}$ is less than $N$. Thus

$$
\sigma^{4}=\sum_{j=1}^{l} \gamma_{j} \quad \text { for some } l \leq N,
$$

where each $\gamma_{j}$ joins boundary components of $D_{n}^{3} \backslash \widehat{O}$. Now, successively, extend the ends of all segments $\gamma_{j}$ horizontally to the left and right until either they first intersect a crossing-boundary in $\mathcal{B}_{n}^{2}$ or otherwise until they meet the next segment $\gamma_{j+1}$. Let $\sigma^{2}$ denote the resulting chain and note that $\sigma^{2} \in \Gamma_{n}^{2}$. It follows from the definition of $\rho_{3}(z)$ that $l_{\rho_{2}}\left(\sigma^{2}\right) \leq l_{\rho_{3}}\left(\sigma^{3}\right)$. Since $L_{\rho_{2}}\left(\Gamma_{n}^{2}\right) \leq l_{\rho_{2}}\left(\sigma^{2}\right)$ and $\sigma^{3} \in \Gamma_{n}^{3}$ was arbitrary, we have that

$$
L_{\rho_{2}}\left(\Gamma_{n}^{2}\right) \leq L_{\rho_{3}}\left(\Gamma_{n}^{3}\right) .
$$

We now consider the area to length-squared ratio. The combination of the inequalities (34) and (35) yields that

$$
\frac{A_{\rho_{2}}\left(D_{n}^{2}, \mathcal{B}_{n}^{2}\right)}{L_{\rho_{2}}\left(\Gamma_{n}^{2}\right)^{2}} \geq \frac{A_{\rho_{3}}\left(D_{n}^{3}, \mathcal{B}_{n}^{3}\right)}{L_{\rho_{3}}\left(\Gamma_{n}^{3}\right)^{2}}-\frac{\frac{\delta}{4} A_{b}\left(\mathcal{B}_{n}\right)+\kappa_{1} \epsilon}{L_{\rho_{2}}\left(\Gamma_{n}^{2}\right)^{2}} .
$$

Since inequalities (32), (27), and (28) imply $L_{\rho_{2}}\left(\Gamma_{n}^{2}\right)>\frac{1}{2} A_{b}\left(\mathcal{B}_{n}\right)^{1 / 2}$, we have

$$
\frac{\frac{\delta}{4} A_{b}\left(\mathcal{B}_{n}\right)+\kappa_{1} \epsilon}{L_{\rho_{2}}\left(\Gamma_{n}^{2}\right)^{2}} \leq \frac{\frac{\delta}{4} A_{b}\left(\mathcal{B}_{n}\right)+\kappa_{1} \epsilon}{\left(A_{b}\left(\mathcal{B}_{n}\right)^{1 / 2} / 2\right)^{2}}=\delta+\kappa_{3} \epsilon
$$

where $\kappa_{3}>0$ is a constant independent of $\epsilon$. Therefore

$$
\frac{A_{\rho_{2}}\left(D_{n}^{2}, \mathcal{B}_{n}^{2}\right)}{L_{\rho_{2}}\left(\Gamma_{n}^{2}\right)^{2}} \geq \frac{A_{\rho_{3}}\left(D_{n}^{3}, \mathcal{B}_{n}^{3}\right)}{L_{\rho_{3}}\left(\Gamma_{n}^{3}\right)^{2}}-\delta-\kappa_{3} \epsilon .
$$

We now apply Theorem 3.3 to the crossing-module problem $\left(D_{n}^{3}, \mathcal{B}_{n}^{3}, \Gamma_{n}^{3}\right)$. We need only remark that application of this theorem is permissible since there are only finitely many horizontal slit components in $\partial D_{n}^{3}$ and all other boundary components, except the outer component, are crossing-boundaries which do not oscillate arbitrarily. The theorem gives that

$$
\mu\left(\Gamma_{n}^{3}\right)=\frac{s-\epsilon}{s+\epsilon} .
$$

By the definition of crossing-module we thus have that

$$
\frac{A_{\rho_{3}}\left(D_{n}^{3}, \mathcal{B}_{n}^{3}\right)}{L_{\rho_{3}}\left(\Gamma_{n}^{3}\right)^{2}} \geq \frac{s-\epsilon}{s+\epsilon} .
$$

Putting together the inequalities (24), (30), (33), (36), and (37), we obtain the following estimate on the original crossing-module ratio:

$$
\frac{A_{\rho}\left(D_{n}, \mathcal{B}_{n}\right)}{L_{\rho}\left(\Gamma_{n}^{N}\right)^{2}} \geq \frac{s-\epsilon}{s+\epsilon}-\delta-\kappa_{3} \epsilon-\kappa_{2} \epsilon .
$$


for any positive number $\epsilon$ less than $\min \left\{1, \epsilon_{0}, \epsilon_{1}, \epsilon_{2}, \epsilon_{3}, \epsilon_{4}\right\}$. Therefore we have shown that

$$
\frac{A_{\rho}\left(D_{n}, \mathcal{B}_{n}\right)}{L_{\rho}\left(\Gamma_{n}^{N}\right)^{2}} \geq 1-\delta
$$

holds for an arbitrary metric $\rho(z)$ on $D_{n}$ satisfying $L_{\rho}\left(\Gamma_{n}^{N}\right)>A_{b}\left(\mathcal{B}_{n}\right)^{1 / 2}$. The combination of this inequality and implication (23) yields the desired result that $\mu\left(\Gamma_{n}^{N}\right) \geq 1-\delta$, and hence that

$$
\mu\left(\Gamma_{n}\right)=1 .
$$

Finally, we show that $f(z)$ achieves $\Theta$. Suppose to the contrary that some component $B^{*} \in \mathcal{B}(f(\Omega))$ were not a slit at the desired inclination $\theta_{j}$. Choose any $\Theta \circ\left(f^{-1}\right)^{\mathcal{C}}$-induced crossing-boundary collection $\mathcal{B}$ and sufficiently large $s$. Define $\left(D_{j}, \mathcal{B}_{j}, \Gamma_{j}\right)$ by $(20)$, and consider the crossing-module problem $\left(D_{j}^{\prime}, \mathcal{B}_{j}^{\prime}, \Gamma_{j}^{\prime}\right)$ induced by the map $z \mapsto e^{-i \theta_{j}} z$. The set $B^{* \prime}=e^{-i \theta_{j}} B^{*}$ is a boundary component of $D_{j}^{\prime}$ which is not a horizontal slit, and satisfies $\operatorname{dist}\left(B^{* \prime}, \mathcal{B}_{j}^{\prime}\right)>0$. Thus, by Theorem 3.6, $\mu\left(\Gamma_{j}^{\prime}\right)<1$. On the other hand, since the crossing-module is invariant under rotations, $\mu\left(\Gamma_{j}^{\prime}\right)=\mu\left(\Gamma_{j}\right)=1$. This contradiction shows that $f(z)$ must achieve $\Theta$.

\section{ACKNOWLEDGMENTS}

We are grateful for communications from Zheng Xu He, Fumio Maitani, David Minda, Burt Rodin, and Oded Schramm.

\section{REFERENCES}

[ABB] J. M. Anderson, K. F. Barth, and D. A. Brannon, Research problems in complex analysis, Bull. London Math. Soc. 9 (1977), 129-162. MR 55:12899

[AS] L. V. Ahlfors and L. Sario, Riemann Surfaces, $2^{\text {nd }}$ ed., Princeton University Press, Princeton, New Jersey 1960. MR 22:5729

[Go] G. M. Goluzin, Geometric Theory of Functions of a Complex Variable, $2^{\text {nd }}$ ed., English Translation, Amer. Math. Soc., Providence, Rhode Island, 1969. MR 40:308

[Gr1] H. Grötzsch, Über das Parallelschlitztheorem der konformen Abbildung schlichter Bereiche, Ber. Verh. sächs Akad. Wiss. Leipzig, Math.-phys. Kl. 84 (1932), 15-36.

[Gr2] H. Grötzsch, Zum Parallelschlitztheorem der konformen Abbildung schlichter unendlichvielfach zussamenhängender Bereiche, Ber. Verh. sächs Akad. Wiss. Leipzig, Math.-phys. Kl. 83 (1931), 185-200.

[Ha] A. N. Harrington, Conformal Mappings on domains with arbitrarily specified boundary shapes, Journal D'analyse Mathématique 41 (1982), 39-53.

[HS] Z.-X. He and O. Schramm, Fixed points, Koebe uniformization and circle packings, Ann. of Math. 137 (1993), 369-406. MR 96b:30015

[J] J. A. Jenkins, Univalent Functions and Conformal Mapping, Springer-Verlag, Berlin, 1958. MR 20:3288

[K] P. Koebe, Abhandlungen zur Theorie der konformen Abbildung: V. Abbildung mehrfach zusammenhängender schlichter Bereiche auf Schlitzbereiche, Math. Z. 2 (1919), 198-236.

[MM] F. Maitani and D. Minda, Rectilinear slit conformal mappings, J. Math. Kyoto Univ. 36 (1996), 659-668. MR 98c:30009

[MR] A. Marden and B. Rodin, Extremal and conjugate extremal distance on open Riemann surfaces with applications to circular-radial slit mappings, Acta Mathematica, Vol. 115 (1966), 237-269. MR 34:2862

[NS] M. Nakai and L. Sario, Classification Theory of Riemann Surfaces, Springer Verlag, New York 1970. MR 41:8660

[dP] R. de Possel, Zum Parallelschlitzentheorem unendlich-vielfach zusammenhängender Gebeite, Nachr. Ges. Wiss. Göttingen, Math.-phys. Kl. (1931), 192-202. 
[RW] E. Reich and S. E. Warschawski, On canonical conformal maps of regions of arbitrary connectivity, Pacific Journal of Mathematics, Vol. 10, No. 3 (1960), 965-985. MR 22:8120

[RS] B. Rodin and L. Sario, Principal Functions, Van Nostrand Co., Inc., Princeton, New Jersey 1968. MR 37:5378

[Sc1] O. Schramm, Conformal Uniformization and Packing, Israel J. Math., 93 (1996), 399-428. MR 96m:52029

[Sc2] O. Schramm, Transboundary extremal length, J. D'Analyse Mathématique, 66 (1995), 307-329. MR 96k:30009

[Sh] M. Shiba, On the Riemann-Roch theorem on open Riemann surfaces, J. Math. Kyoto Univ., 11 (1971), 495-525. MR 45:536

[W] F. Weening, Existence and Uniqueness of Non-parallel Slit Maps, Ph. D. dissertation, 1994.

Department of Mathematics, University of California at San Diego, La Jolla, CalIFORNIA 92093

E-mail address: cfitzgerald@ucsd.edu

Department of Mathematics, University of California at San Diego, La Jolla, CalIFORNIA 92093

Current address: Department of Mathematics and Computer Science, Doucette Hall, Edinboro University of Pennsylvania, Edinboro, Pennsylvania 16444

E-mail address: fweening@edinboro.edu 